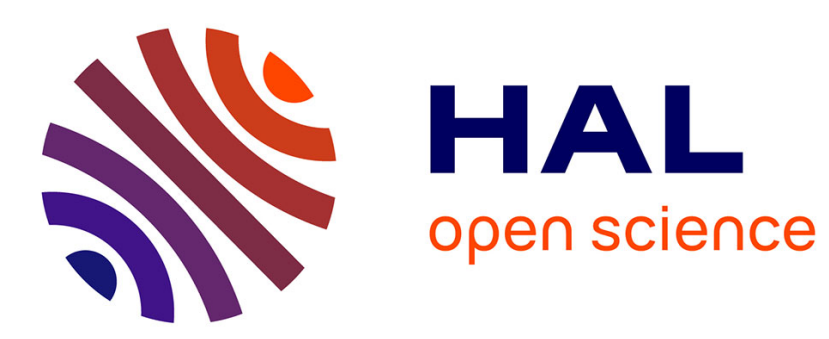

\title{
Fiber orientation effects on heat source distribution in reinforced polyamide 6.6 subjected to low cycle fatigue
}

\author{
Adil Benaarbia, André Chrysochoos, Gilles Robert
}

\section{To cite this version:}

Adil Benaarbia, André Chrysochoos, Gilles Robert. Fiber orientation effects on heat source distribution in reinforced polyamide 6.6 subjected to low cycle fatigue. Journal of Engineering Mathematics, inPress, 90 (1), pp.13-36. 10.1007/s10665-014-9720-7 . hal-01095173

\section{HAL Id: hal-01095173 \\ https://hal.science/hal-01095173}

Submitted on 17 Dec 2014

HAL is a multi-disciplinary open access archive for the deposit and dissemination of scientific research documents, whether they are published or not. The documents may come from teaching and research institutions in France or abroad, or from public or private research centers.
L'archive ouverte pluridisciplinaire HAL, est destinée au dépôt et à la diffusion de documents scientifiques de niveau recherche, publiés ou non, émanant des établissements d'enseignement et de recherche français ou étrangers, des laboratoires publics ou privés. 


\title{
Fiber orientation effects on heat source distribution in reinforced polyamide 6.6 subjected to low cycle fatigue
}

\author{
Adil Benaarbia - André Chrysochoos · Gilles Robert
}

\begin{abstract}
The present study was aimed at gaining further insight into the behavior of short fiber-reinforced semicrystalline-polymeric (SFRSP) composites conditioned at atmospheric pressure with air containing $50 \%$ relative humidity and subjected to repetitive loadings. The experimental approach was based on synchronized full-field temperature and strain data to investigate and characterize the thermomechanical response of these heterogeneous materials throughout tensile-tensile oligocyclic loading tests. The overarching aim was to determine the thermomechanical constitutive properties of SFRSP composites over a range of fiber orientation angles, for example, $0^{\circ}, 45^{\circ}$, and $90^{\circ}$. The thermomechanical behavior of a dry polyamide 6.6 matrix was also analyzed in a previous study using the same experimental approach. This approach involved digital image correlation (DIC) and infrared thermography (IRT). The first was used to track random surface pattern movements to monitor displacement or deformation, while the second recorded in-plane temperature changes from which the amount of heat sources involved during the material deformation was derived. Noticeable in-plane heterogeneities and the effects of fiber orientation on heat-source patterns, for example, intrinsic dissipation and thermoelasticity ranges, were highlighted and examined using these imaging techniques. The energy content of hysteresis loops was identified, and no elastic or plastic shakedown was noted, so there was no real cyclic adaptation. The heat conversion factor, i.e., Taylor-Quinney coefficient, was also calculated to quantify the relative contribution of stored and dissipated energies in the energy balances of the SFRSP composites.
\end{abstract}

Keywords Digital image correlation - Dissipated energy rate - Fiber orientation angle effect · Infrared thermography · Taylor-Quinney ratio · Thermoelasticity · Short fiber-reinforced semicrystalline-polymeric composites

\footnotetext{
A. Benaarbia $(\varangle) \cdot$ A. Chrysochoos

University Montpellier 2, LMGC Laboratory, CNRS, Place Eugène Bataillon, 34095 Montpellier, France

e-mail: abenaarb@univ-montp2.fr
}

A. Chrysochoos

e-mail: andre.chrysochoos@univ-montp2.fr

G. Robert

Solvay Engineering Plastics, Technyl Innovation Center-Simulation et Validation des Applications, Avenue Ramboz, BP 64, 69192 Saint Fons, France

e-mail: Gilles.ROBERT@ solvay.com 


\section{Introduction}

Owing to their great thermomechanical advantages, fiber-reinforced polymeric materials are widely used in environments with high temperature fluctuations and complex loadings. Considering the recent growing interest in these engineering materials for automobile structures, it is essential to enhance the overall understanding of their thermomechanical constitutive behavior during repetitive cyclic loadings. Though the behavior of such materials subjected to tensile loading is reasonably well understood, the response to complex loadings requires further investigation [1,2]. Analysis of the mechanical, thermal, and energy properties of these composite materials is complicated because of the many parameters involved, for example, the matrix composition, type of reinforcement (short or long fibers), and the fiber architecture. For example, to achieve successful modeling of the behavior of these heterogeneous materials under energy considerations, the fiber orientation and its effects on the overall structural performance must be clarified. There is therefore a need to gain some insight into the change in fiber orientation and to determine certain relationships between the fiber orientation and locally observed dissipative mechanisms. For example, prediction of stress and dissipation singularities at the level of the fibers and surrounding matrix can facilitate detection of areas susceptible to local failure. The intensity of these singularities strongly depends on the mechanical and thermal properties of the loaded materials.

Complete energy balances associated with the material deformation must be determined to enable the collection of further information on these interesting local dissipative phenomena. Otherwise, drawing up energy balances may provide greater insight into the evolution of the physical phenomena that cause temperature increases (or decreases) observed when polymeric composites are subjected to cyclic loadings. Many experimental, theoretical, and numerical studies still focus on determining such energy balances. When a material is subjected to anelastic transformations, part of the energy expended in deformation is dissipated (i.e., irreversibly transformed into heat), but the rest remains stored in the material, thereby modifying its internal energy. This stored energy is undoubtedly associated with changes in the physical material properties due to microstructural transformations. Many interesting surveys on specific aspects of the stored energy can be found in the literature. Several research studies using calorimetric techniques have focused on the determination of changes in temperature increases during loadings [3-7]. However, for repetitive loading, few previous investigations have been carried out on the measurement of stored energy in polymeric materials. Most of the investigations were undertaken largely in tensile mode [8-10].

Furthermore, it is essential to identify the energy content of hysteresis loops in order to evaluate the material damage during cyclic processes. Dissipative mechanisms are generally responsible for the mechanical energy lost in the stress-strain loop, reflecting irreversible material degradation. These mechanisms can be induced by viscous or viscoplastic deformation processes or by damage growth [11]. However, thermodynamic analysis of the cyclic responses revealed that such hysteresis areas may be induced by intrinsic dissipation and by internal energy variations (stored energy) or by strong thermomechanical coupling effects associated with heat diffusion [12].

Otherwise, the heat produced by deformation is generally obtained from the measured increase (or decrease) in temperature of the specimen, its heat capacity, and heat losses. Calorimetric techniques can be used to derive heat sources from thermal data. Infrared thermography (IRT) is a suitable technique for obtaining thermal images from which the dissipated energy value can be determined using the heat diffusion equation, which is a direct combination of the first and second principles of thermodynamics. The stored energy can thus be obtained as the difference between the deformation energy of the sample and the heat evolved by it. The deformation energy is determined from local stress-strain data. The digital image correlation gives access to in-plane displacement components. Using spatiotemporal derivatives applied to these in-plane displacement data, strain and strain rate tensors can then be obtained. Both imaging techniques can thus be combined to establish energy balances [13].

This paper is a further development of previous studies that primarily dealt with local hot spot detection in dry polyamide (PA) 6.6 matrix specimens [14]. Although we used the same theoretical framework and experimental approach for the same polymeric material, the comparison of the results obtained with a dry PA6.6 matrix and those obtained with a reinforced PA6.6 conditioned at a different relative humidity would be difficult and premature, mainly because the matrix behavior is highly dependent on the relative humidity (water plasticizing effect). For instance, current experiments on PA6.6 specimens conditioned at $50 \%$ relative humidity suggest that the deformation 
energy rate and the associated energy dissipation for the same loading conditions are respectively 20 -fold and 60 fold lower for the dry matrix than those obtained with the $\mathcal{R} \mathcal{H} 50$ matrix. For this reason, we only focus on the thermomechanical analysis of SFRSP at $\mathcal{R H} 50$ in what follows.

To fulfill the primary objective of the present survey, a brief description of independent heat sources, dissipation, and thermoelasticity is first presented, followed by the theoretical background used to interpret the experimental findings. Then thermal and deformation measurement methods are proposed and discussed in detail. These methods have brought further insight into the dissipation and stress localization fields in the investigated specimens. Different fiber configurations of the investigated composite materials were systematically considered: $0^{\circ}, 45^{\circ}$, and $90^{\circ}$. The fiber orientation effects in these composite materials are then underlined and discussed. Local energy balances associated with the low cycle fatigue of these composites are established, as is the relative contribution of several mechanical energy components.

\section{Thermal observations in fatigue process}

A material undergoing mechanical loading often dissipates a part of the deformation energy as heat. This dissipated part generates a progressive increase in material temperature variations. These thermal variations are not intrinsically related to the material behavior because they are affected by heat transfers. Therefore, there is a strong need to find alternative ways to evaluate more intrinsic data representative of the material behavior, for example, heat sources. These sources are directly connected to constitutive laws of the material behavior. To this end, techniques of infrared (IR) image processing were developed to evaluate heat sources accompanying the deformation process [15-17]. One promising way to assess these heat phenomena involves the use of temperature measurements. Temperature is an observable state variable that has been adopted in numerous approaches to model the material behavior in an anisothermal thermodynamic context.

Changes in material temperature are the result of two fundamentally independent heat sources: coupling sources and dissipation. Coupling sources characterize the material thermodilatability, i.e., a temperature drop during loading and a temperature rise during unloading. They are reflected by oscillations in opposite phase with the monochromatic loading signal (Fig. 1a). The second heat source is attributed to the irreversibilities that accompany the deformation of the material, i.e., so-called dissipation. For adiabatic conditions, this dissipative heat source generates a monotonic increase in temperature variations during loading. From a calorimetric standpoint, it was already found that intrinsic dissipation intensities remained very low compared with the thermoelastic source amplitude ranges for dry PA6.6 matrix specimens [14].

For in-plane stress conditions, the reversible change in temperature $\Delta \theta$ is supposed to be proportional to the change in the first stress invariant, as shown by Thomson [18] and recently discussed in [16]. This relationship is commonly formulated under adiabatic conditions as follows:

$\Delta \sigma_{I}=\xi T_{o} \Delta \theta$,

where $\Delta \sigma_{I}$ denotes the change in the first invariant of the stress tensor, $\theta$ represents the temperature variations, i.e., $\theta=T-T_{o}$, with $T$ and $T_{o}$ respectively the current and room temperature. $\xi=-\rho C / \alpha$ stands for the thermoelastic coupling constant, where $\alpha$ represents the coefficient of thermal expansion, and $\rho$ and $C$ are respectively the material density and heat capacity.

In Fig. 1b, we can distinguish two stages in the fatigue life of a typical short fiber-reinforced semicrystallinepolymeric (SFRSP) specimen until final rupture. These stages are characterized by their own thermal effects: the first stage shows a gradual rise in the average specimen temperature due to an increase in anelastic strain (ratcheting effect). The second stage corresponds to a sharp increase in temperature due to viscoelastic strain and damage localization effects, rapidly leading to the macroscopic crack as the curve at its right extremity shows in Fig. $1 \mathrm{~b}$. 
(a) $\widehat{0}$

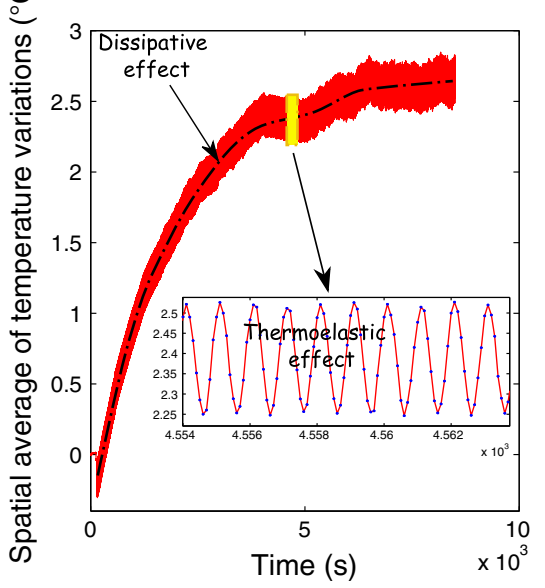

(b)

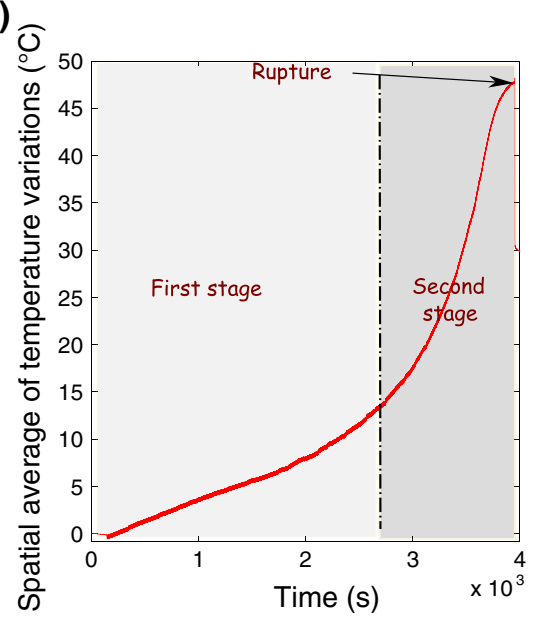

Fig. 1 a Typical time course of temperature variations $\langle\theta\rangle$ associated with $\mathrm{MGF} / 45^{\circ}$ conditioned at $50 \%$ relative humidity, subjected to a $10 \mathrm{~Hz}$ loading fatigue test showing both heat source effects: intrinsic dissipation and thermoelasticity. $\mathbf{b}$ Typical time course of $\langle\theta\rangle$ until final crack, in a $\mathrm{MGF} / 45^{\circ}$ specimen conditioned at $50 \%$ relative humidity, subjected to a $10 \mathrm{~Hz}$ loading frequency, with a stress ratio of 0.1 . The chosen stress range was greater in $\mathbf{b}$ than in $\mathbf{a}$ so that the specimen rupture would be achieved before the end of the cyclic test

\section{Theoretical background}

The framework of the thermodynamics of irreversible processes (TIP) provides expressions for how the internal energy changes during a deformation process [19]. Under the small deformation hypothesis, the first principle of thermodynamics can be written as [20]

$\rho \dot{e}=\sigma: \dot{\varepsilon}+r_{e}-\operatorname{div} \boldsymbol{q}$,

where $\rho$ denotes the material density, $\dot{e}$ the rate of specific internal energy, $\sigma$ the Cauchy stress tensor, $\dot{\boldsymbol{\varepsilon}}$ the strain rate tensor, $r_{e}$ an external heat supply that characterizes the volume heat exchange with the surroundings, and $\boldsymbol{q}$ the heat influx vector. Irreversible processes are conventionally defined from the second principle of thermodynamics. This principle is expressed as the generalized Clausius-Duhem inequality, assuming that the rate of entropy production cannot be negative. In this context, a local expression for the second law of thermodynamics can be formulated as

$\rho \dot{s} \geq T^{-1}\left(r_{e}-\operatorname{div} \boldsymbol{q}+\frac{\boldsymbol{q}}{T} \cdot \operatorname{grad} T\right)$,

where $T=\partial e / \partial s$ stands for the absolute temperature and $\dot{s}$ is the rate of specific entropy of the material.

By combining both of the foregoing expressions for the first and second principles, Eqs. (2) and (3), we recall in the next subsection the classical heat diffusion equation and its operational form specially adapted for estimating dissipative mechanisms that cause irreversibilities in the material state.

\subsection{Heat diffusion equation}

According to the local state axiom [20], the thermodynamic state of a material is described by a finite set of state variables. These variables include observable and so-called internal variables. The observable variables are generally the absolute temperature $T$ and the overall strain tensor $\boldsymbol{\varepsilon}$. The internal variables, denoted here by the 
set $\left\{\alpha_{k}\right\}_{k=2, \ldots, m}$, are representative of the microstructural features. The choice of internal variables depends on the spatiotemporal scales at which the modeling is performed.

In the generalized standard materials formalism [19], any material behavior can be summed up in two potentials: thermodynamic potential $\psi$ and dissipation potential $\varphi$. The thermodynamic potential, classically considered Helmholtz free energy $\psi=e-T s$, is a state function. Its partial derivatives with respect to the state variables are the so-called state equations, i.e., relations between the state variables and their conjugated variables. The thermomechanical properties of equilibrium states can be completely described using this thermodynamic potential. Irreversiblities associated with deformation processes are introduced via the dissipation potential, which is a function of the state variable rates $\dot{\boldsymbol{\varepsilon}}, \dot{\alpha}_{k}$, and $\boldsymbol{q}$. The partial derivatives of this dissipation potential with respect to the state variable rates give the so-called evolution (or complementary) equations, i.e., relations between the state variable rates vector and its associated vector of thermodynamic forces $\left(\sigma-\rho \partial \psi / \partial \boldsymbol{\varepsilon},-\rho \partial \psi / \partial \alpha_{k},-T^{-1} \operatorname{grad} T\right)$. These thermodynamic forces derive from the total dissipation $\mathcal{D}$ (mechanical version of Clausius-Duhem inequality) classically split into mechanical (intrinsic) $\mathcal{D}_{1}$ and thermal dissipation $\mathcal{D}_{2}$ (Eq. 4) (readers interested in the continuum thermodynamics and the thermodynamic formulation of constitutive equations are referred to [21-23]):

$\mathcal{D}=\underbrace{\left(\sigma-\rho \frac{\partial \psi}{\partial \boldsymbol{\varepsilon}}\right): \dot{\boldsymbol{\varepsilon}}-\rho \frac{\partial \psi}{\partial \alpha_{k}} \dot{\alpha}_{k}}_{\mathcal{D}_{1}}-\underbrace{\frac{\boldsymbol{q}}{T} \cdot \operatorname{grad} T}_{\mathcal{D}_{2}} \geq 0$.

Note that the dissipation can be written as a sum of products of thermodynamic forces and fluxes of state variables. Naturally, the product symbol changes with the tensoriality order of the state variable flux and of its associated thermodynamic forces. Let us define the specific heat as $C_{\boldsymbol{\varepsilon}, \alpha}=-T \partial^{2} \psi / \partial T^{2}$ at constant $\boldsymbol{\varepsilon}$ and $\alpha_{k}$, and let $\boldsymbol{k}$ denote the heat conduction tensor. In reference to the details provided in the studies [24,25], where more references on the development of the heat theory can also be found, here we recall the local expression of the heat diffusion equation:

$\rho C_{\boldsymbol{\varepsilon}, \alpha} \dot{T}+\operatorname{div}(\underbrace{-\boldsymbol{k} \operatorname{grad} T}_{\boldsymbol{q}})=\underbrace{\left(\boldsymbol{\sigma}-\rho \frac{\partial \psi}{\partial \boldsymbol{\varepsilon}}\right): \dot{\boldsymbol{\varepsilon}}-\rho \frac{\partial \psi}{\partial \alpha_{k}} \dot{\alpha}_{k}}_{\mathcal{D}_{1}}+\underbrace{\rho T \frac{\partial^{2} \psi}{\partial T \partial \boldsymbol{\varepsilon}}: \dot{\boldsymbol{\varepsilon}}+\rho T \frac{\partial^{2} \psi}{\partial T \partial \alpha_{k}} \dot{\alpha}_{k}}_{s_{c}}+r_{e}$.

This expression equates thermal data, which can be experimentally provided by the IR camera, with heat sources that arise during material deformation. The left-hand side of Eq. (5) expresses the thermal inertia $\rho C_{\varepsilon, \alpha} \dot{T}$, which represents the stored or released heat rate and $\operatorname{div}(-k \operatorname{grad} T)$, which is the volume heat lost by conduction. The right-hand side, in turn, includes the intrinsic mechanical dissipation $\mathcal{D}_{1}$, which reflects the irreversible material transformations, and the thermomechanical coupling sources $s_{c}$, which are the calorific signatures of possible interactions between the temperature and the other state mechanical or microstructural variables.

For the sake of simplicity, we often simplify the preceding local heat diffusion equation to more easily perform the heat source assessment. The experimental conditions of our tests are based on the following hypothesis:

- $\left(\mathcal{H}_{1}\right)$ The thermophysical characteristics $\left(\rho, \boldsymbol{k}, C_{\boldsymbol{\varepsilon}, \alpha}\right)$ are considered material constants independent of the thermodynamic state ( $\boldsymbol{k}$ is also assumed to be isotropic, i.e., $\boldsymbol{k}=k \boldsymbol{\delta}$, where $\boldsymbol{\delta}$ is the identity tensor and $k$ the isotropic conduction coefficient). An isotropy hypothesis of heat conduction is naturally questionable for heterogeneous, anisotropic composite materials. However, the conduction coefficient of the matrix is here approximately $0.34 \mathrm{Wm}^{-1} \mathrm{~K}^{-1}$ for a heat diffusion coefficient of approximately $2 \times 10^{-7} \mathrm{~m}^{2} \mathrm{~s}^{-1}$, corresponding to those of some thermal insulators. In addition, the conduction coefficient of glass fibers is tenfold lower $\left(0.03 \mathrm{Wm}^{-1} \mathrm{~K}^{-1}\right)$ compared to that of the matrix. Thus, the heat diffusion in the reinforced composites essentially takes place in the matrix. In addition, considering the radius $\left(r_{f}=5 \mu \mathrm{m}\right)$ and the heat diffusion coefficient of the fibers $\left(D_{f}=1.4 \times 10^{-9} \mathrm{~m}^{2} \mathrm{~s}^{-1}\right)$, the time characterizing the heat diffusion in these fibers is approximately $r_{f}^{2} / D_{f} \approx 20 \mathrm{~ms}$, suggesting a quasi-instantaneous thermal equilibrium between the fibers and the matrix. This 
analysis led us to consider that the fibers did not markedly modify the heat diffusion mechanisms, and so SFRSP composites were assumed to behave as isotropic materials from a thermal standpoint.

- $\left(\mathcal{H}_{\mathbf{2}}\right)$ The heat exchange $r_{e}$, here by radiations, is assumed to be time independent. Consequently, the equilibrium temperature field $T_{o}$ authenticates $-k \Delta T_{o}=r_{e}$, where $\Delta$ stands for the Laplacian operator.

- $\left(\mathcal{H}_{3}\right)$ As long as the displacement velocity amplitudes and temperature gradients remain low, the convective terms in the total time derivative of the temperature may also be neglected.

- $\left(\mathcal{H}_{4}\right)$ As temperature variations induced by the fatigue process remain small, we assume that they have no interaction with the microstructural state. This means that $\rho T \psi_{, T, \alpha_{k}} \dot{\alpha}_{k} \approx 0$, and thus only the thermoelastic coupling effects are considered.

- $\left(\mathcal{H}_{5}\right)$ For thin flat specimens, the surface temperature is representative of the temperature in the thickness [26]. The measured temperature field can thus be written

$\bar{T}(x, y, t)=T_{I R}(x, y, t)=\frac{1}{e} \int_{-e / 2}^{e / 2} T(x, y, z, t) \mathrm{d} z$,

where $\{x, y, z\}$ denote the set of the Eulerian coordinates and $t$ the time, respectively.

Considering the foregoing hypotheses, it is useful to reduce the generalized local heat diffusion equation, Eq. (5), to the following two-dimensional thermal diffusion model:

$\frac{\partial \bar{\theta}}{\partial t}+\frac{\bar{\theta}}{\tau_{\text {th }}^{2 \mathrm{~d}}}-\frac{k}{\rho C}\left(\frac{\partial^{2} \bar{\theta}}{\partial x^{2}}+\frac{\partial^{2} \bar{\theta}}{\partial y^{2}}\right)=\frac{\overline{\mathcal{D}}_{1}}{\rho C}+\frac{\bar{s}_{\text {ther }}}{\rho C}$,

where $\bar{\theta}=\bar{T}-\bar{T}_{o}, \overline{\mathcal{D}}_{1}$, and $\bar{s}_{\text {ther }}$ represent the temperature variations, intrinsic dissipation, and thermoelastic sources averaged over the sample thickness, respectively, whereas the term $\tau_{\text {th }}^{2 \mathrm{~d}}$ stands for the time constant characterizing perpendicular heat exchanges between the outer in-plane surfaces of the specimen and the surroundings.

\subsection{Energy balance}

The mechanical energy per volume unit mechanically lost during material deformation is determined by computing the area of the hysteresis loop enclosed in the stress-strain loop. It can be shown that the mechanical energy over a complete cycle $\left\langle\tilde{\mathcal{W}}_{\text {def }}\right\rangle$ can be split into three parts, i.e., thermoelastic, stored, and dissipated energies. While thermoelastic and dissipated energies are introduced in Sect. 3.1, the other one (stored energy) $\left\langle\mathcal{W}_{s}\right\rangle$ depicts the internal energy changes induced by anelastic transformations. An essential feature of this stored energy is that it remains in the deformed material, at least temporarily, until possible unloading or restoration processes. For one complete load-unload cycle, the stored energy amounts, averaged over this cycle, are obtained as the difference between the deformation energy spent during the material transformation and the dissipated energy. In this context, the stored energy rate averaged over a complete cycle $\left\langle\tilde{\mathcal{W}}_{s}\right\rangle$ reads

$\left\langle\tilde{\dot{\mathcal{W}}}_{s}\right\rangle=\left\langle\tilde{\dot{\mathcal{W}}}_{\text {def }}\right\rangle-\left\langle\tilde{\mathcal{D}}_{1}\right\rangle$

The operator $\langle\tilde{\mathbf{x}}\rangle$ denotes the spatiotemporal average of $\mathbf{x}$ over the volume element and the load-unload cycle under consideration.

For clarity, possible cases of energy balances are introduced and analyzed hereinafter. The more general form of the energy balance equation that relates the deformation energy to the various energies involved during a loadunload cycle can be found in [12]. By combining Eqs. (2) and (3), the preceding equation can be generalized even further to the following expression of the volume deformation energy associated with the load-unload test [27]:

$\left\langle\mathcal{W}_{\mathrm{def}}\right\rangle=\int_{t_{\mathcal{A}}}^{t_{\mathcal{B}}}\left\langle\mathcal{D}_{1}\right\rangle \mathrm{d} t+\int_{t_{\mathcal{A}}}^{t_{\mathcal{B}}}\langle\rho \dot{e}-\rho C \dot{T}\rangle \mathrm{d} t+\int_{t_{\mathcal{A}}}^{t_{\mathcal{B}}}\left\langle s_{c}\right\rangle \mathrm{d} t$, 


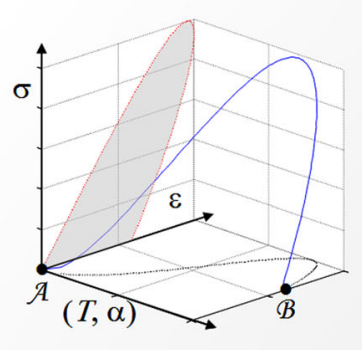

(i) $\mathcal{A} \neq B$

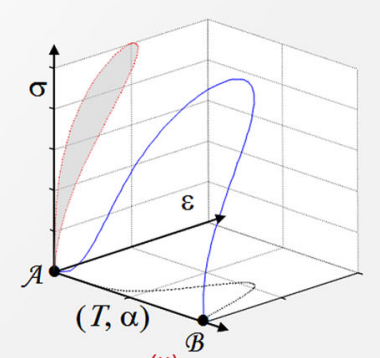

(ii) $\varepsilon_{\mathcal{A}}=\varepsilon_{\mathscr{B}}$

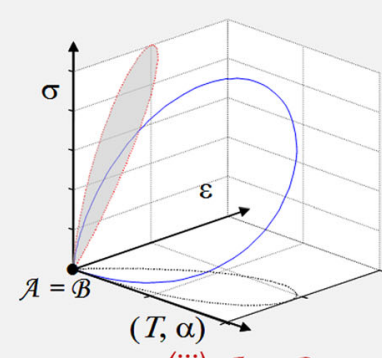

(iii) $A=B$

Fig. 2 Stress-strain diagrams for a load-unload test showing the three possible energy balance situations: $i$ general case, $i i$ mechanical hysteresis loop, and iii thermodynamic cycle [27]

where $\mathcal{A}$ and $\mathcal{B}$ are respectively the thermodynamic states of a material point at the beginning and end of loading.

At first sight, it would seem possible to look at the three basic energy balance situations (Fig. 2). The first case represents a general case where thermodynamic states $\mathcal{A}$ and $\mathcal{B}$ are not identical. In this situation, the mechanical energy mechanically lost during the load-unload cycle can be induced by dissipated energy, internal energy variations, or thermomechanical couplings. In the second case, the strain in $\mathcal{B}$ is equal to that in $\mathcal{A}$, and the load-unload cycle is a mechanical cycle and the area enclosed in the stress-strain hysteresis loop provides an estimate of the mechanical energy value mechanically lost in the transformation. In this case, possible mechanical energy transformations are still similar to those of the first case. In the last case, the mechanical cycle becomes a thermodynamic cycle and the mechanical hysteresis area is exclusively derived from the dissipated energy and thermomechanical couplings.

\section{Experimental analysis}

\subsection{Test specimens}

The experiments were carried out with injection-molded composite plates of PA6.6 (glass transition at equilibrium with air having $50 \%$ relative humidity $\approx 32^{\circ} \mathrm{C}$ and the melting point range $\approx 263^{\circ} \mathrm{C}$ ) reinforced with an E-glass fiber content of $30 \%$ by weight. The composite plates were provided by Solvay Engineering Plastics and are commercially available under the trade name $A 218 \mathrm{~V} 30$. Test specimens were cut from these plates as required (suitable size and shape for the clamping jaws of the loading machine). The dimensions of the machined specimens were $100 \mathrm{~mm} \times 20 \mathrm{~mm} \times 3.14 \mathrm{~mm}$. During cutting, various specimen fiber configurations were manipulated with respect to the loading direction and designated as $\mathrm{MGF} / 0^{\circ}, \mathrm{MGF} / 45^{\circ}$, and $\mathrm{MGF} / 90^{\circ}$ for the fiber orientation angles $0^{\circ}, 45^{\circ}$, and $90^{\circ}$, respectively. The configurations of the composite samples with different orientation angles are shown in Fig. 3. The thermophysical properties of the investigated composites are given in Table 1.

The composites were reinforced with short E-glass fibers to provide local strengthening. Choosing the fiber length as short as possible gives the fibers less surface area, and consequently there will likely be fewer flaws propagated during mechanical loading. Surface treatments resulting from chemical reactions were performed to enhance bonding between the composite constituents. These provide protection for fiber surfaces and minimize degradation of the mechanical properties of the fibers.

\subsection{Failure mode}

Typical fracture modes associated with the investigated composites are presented in Fig. 4. From top to bottom, the fiber orientations are respectively $0^{\circ}, 45^{\circ}$, and $90^{\circ}$. Obviously, we can match the fiber orientation angles with the 


\section{(a)}
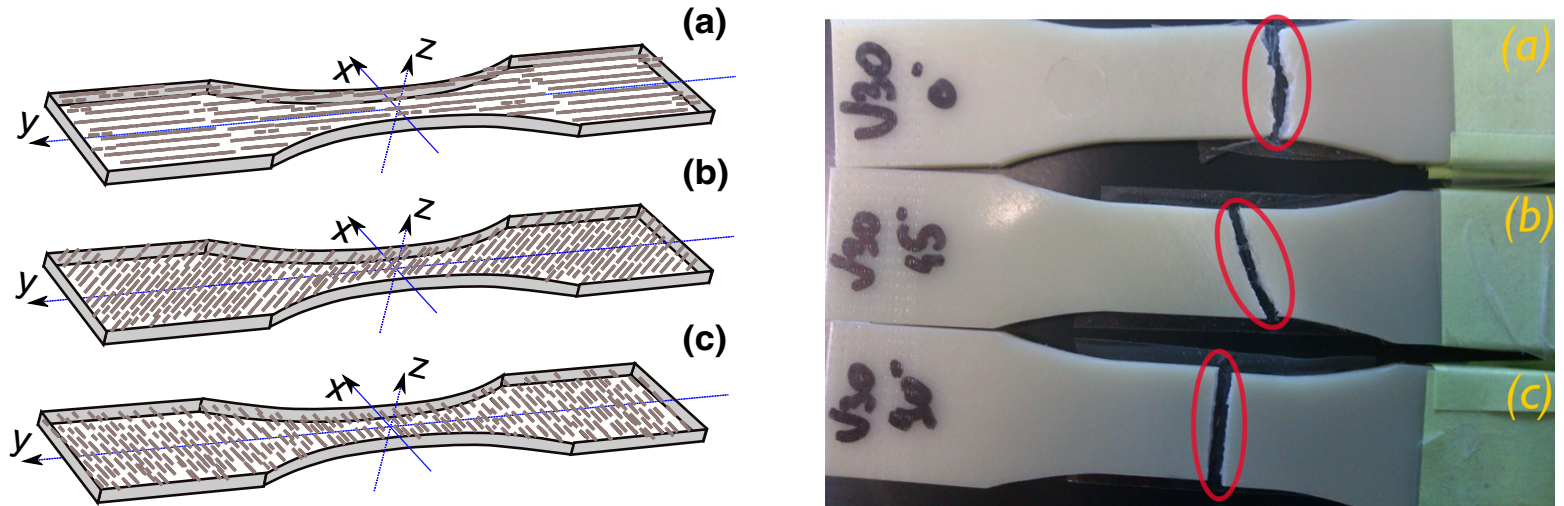

Fig. 3 Sketch of composite sample configurations at different fiber orientation angles for a $\mathrm{MGF} / 0^{\circ}, \mathbf{b} \mathrm{MGF} / 45^{\circ}$, and c MGF $/ 90^{\circ}$

Fig. 4 Fracture mode of composite specimens for a $\mathrm{MGF} / 0^{\circ}$, b $\mathrm{MGF} / 45^{\circ}$, and $\mathbf{c m G F} / 90^{\circ}$

Table 1 Thermophysical properties of studied materials

\begin{tabular}{lllllc}
\hline Material & $\begin{array}{l}\text { Material density } \\
\left(\mathrm{Kg} / \mathrm{m}^{3}\right)\end{array}$ & $\begin{array}{l}\text { Heat capacity } \\
(\mathrm{J} / \mathrm{kg} / \mathrm{K})\end{array}$ & $\begin{array}{l}\text { Mean expansion } \\
\text { coefficient } \\
\left(10^{-6} \mathrm{~K}^{-1}\right)\end{array}$ & $\begin{array}{l}\text { Thermal conduc- } \\
\text { tivity }(\mathrm{W} / \mathrm{K} / \mathrm{m})\end{array}$ & $\begin{array}{l}\text { Mean thermal dif- } \\
\left.\text { fusivity }(\mathrm{mm})^{2} / \mathrm{s}\right)\end{array}$ \\
\hline PA6.6 matrix & $1120 \pm 30$ & $1638 \pm 28$ & $98 \pm 13$ & $0.343 \pm 0.002$ & $0.210 \pm 0.004$ \\
MGF $/ 0^{\circ}$ & $1370 \pm 30$ & $1610 \pm 42$ & $52 \pm 13$ & $0.392 \pm 0.002$ & $0.242 \pm 0.007$ \\
MGF $/ 45^{\circ}$ & $1370 \pm 30$ & $1613 \pm 63$ & $49 \pm 13$ & $0.397 \pm 0.003$ & $0.247 \pm 0.009$ \\
MGF/90 & $1370 \pm 30$ & $1629 \pm 28$ & $76 \pm 13$ & $0.400 \pm 0.004$ & $0.246 \pm 0.008$
\end{tabular}

The thermophysical data were determined using different experimental equipment. The expansion coefficient was measured using a Netzsch Dil 402 C dilatometer, while the other thermal properties were assessed using a Hot Disk thermal analyzer

fracture path. The crack propagates along the fiber direction except for the $0^{\circ}$, in which the fracture initiates in a small area and then extends unstably throughout the whole width of the specimen.

\subsection{Experimental setup and data processing}

The experimental setup used for conducting the thermomechanical tests is shown in Fig. 5. Composite samples were loaded using an MTS 810 hydraulic testing machine equipped with a load cell of $\pm 25 \mathrm{kN}$. Temperature variations on one side and displacements on the other side of the sample were measured and captured simultaneously using both a high-resolution IR focal plane array camera and a high-resolution visible charge-coupled device (CCD) camera, respectively.

IRT is a convenient noncontact imaging technique that uses an IR sensor to visualize and estimate the temperature fields of material surfaces. The principle of the technique is based on the use of the intensity and wavelength of radiation emitted by the loaded material to measure its surface temperature according to Planck's law.

The IR thermal imaging system used for thermographic measurement was a CEDIP Jade camera, cooled by a Stirling cooler. This contained an InSb detector that is sensitive to IR radiation in the 3-5 $\mu \mathrm{m}$ wavelength range. The camera was equipped with a $25 \mathrm{~mm}$ lens and positioned on a tripod approximately $0.65 \mathrm{~m}$ from the specimen surface. We decided to operate the detector with a $160 \times 128$ pixel window size, $29 \mu \mathrm{m}$ pixel size, and $400 \mu \mathrm{s}$ integration time. The camera frame rate was set at ten times the loading frequency to obtain enough sampled data 


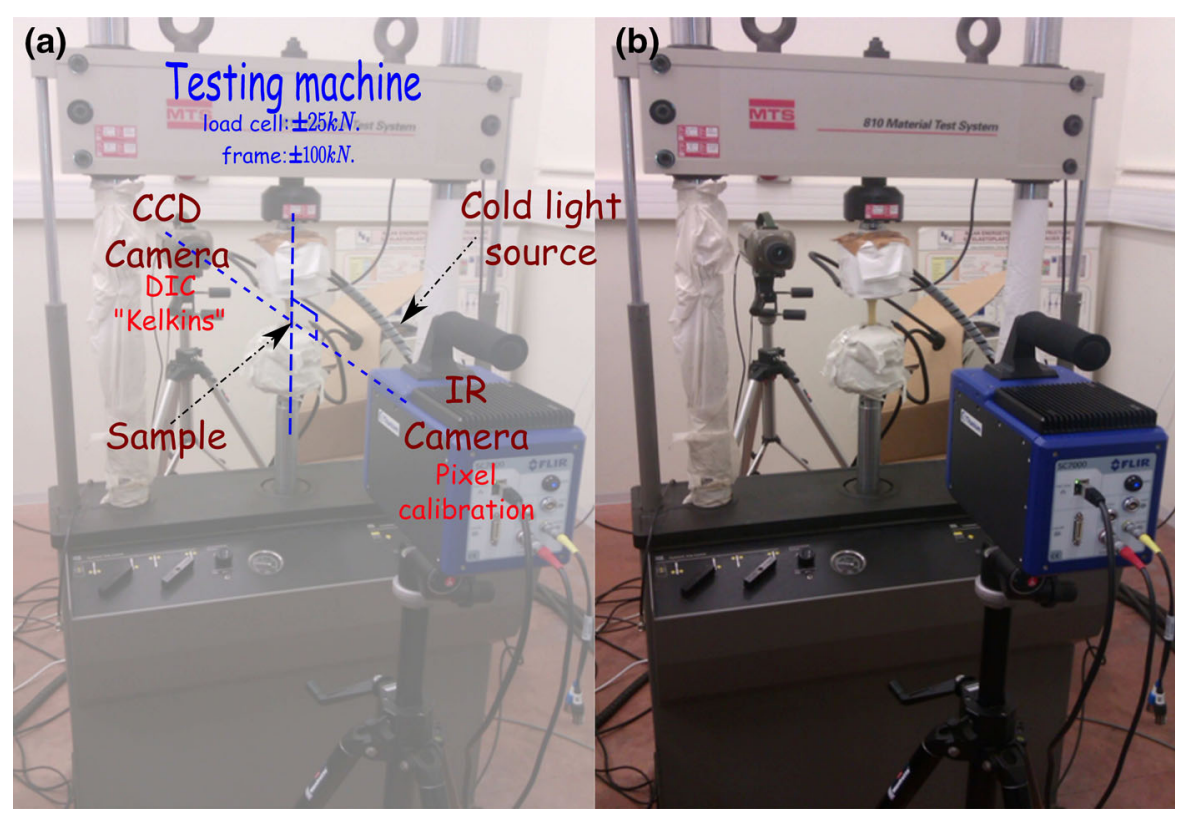

Fig. 5 Experimental setup showing both cameras used for recording displacement and temperature surface fields

to improve the signal-to-noise ratio using local least-squares approximation. Thus, the loading frequency was set at $10 \mathrm{~Hz}$, and the frame rate was set at 100 frames per second, i.e., $100 \mathrm{~Hz}$.

During the experiments, several factors complicated the measurement of the surface temperature of the investigated materials. For this reason, several experimental precautions were taken to overcome these difficulties. For instance, the heat flux density transferred to the IR sensor included noise components because of the reflected incident energy from the surroundings. The IR measurements were therefore made indoors, and the experimental tools were screened and isolated far from radiation sources of the surroundings. In addition, the camera was turned on for $4 \mathrm{~h}$ before starting the experiments in order to reach thermal equilibrium. The temperature was quantitatively measured after improving the surface emissivity. The tested specimens were coated with matt black paint, assuming that the specimens were perfect black bodies with an emissivity value close to 1 . Even though these precautions were taken, the measured data were not necessarily quantitative, and the true temperature value could not be derived directly. An appropriate calibration protocol was thus conducted to determine the true temperature value. This allowed conversion of the thermal radiation digitized by the IR camera into temperature using pixel calibration functions [28]. This pixel protocol was based on a polynomial fitting of the digital levels $\mathcal{D} \mathcal{L}_{(m, n)}$ delivered by each individual detector element $(m, n)$ using a black body with a uniform high emissivity coating, maintained at cold and hot temperatures $T_{(m, n)}$. The measurement accuracy could thus be assessed for each pixel individually in the resulting image:

$T_{(m, n)}=\sum_{k=0}^{d_{\mathrm{f}}} \kappa_{(m, n){ }_{k}} \mathcal{D} \mathcal{L}_{(m, n)}^{\mathrm{k}}$,

where $d_{\mathrm{f}}$ denotes the degree of the polynomial fitting function and $\kappa_{(m, n)_{k}}$ are coefficients of the calibration law for the element $(m, n)$. These coefficients were derived from least-squares fitting approximations.

Two bad-pixel-detection criteria were used. The first consisted of the elimination of pixels whose response predicted by the polynomial fitting $T_{(m, n)}$ was far from the ordered temperature of the used black body $T_{b b}$, whereas the second eliminated pixels whose thermal response was too far from the calculated average temperature throughout the detector $T_{a v}$. In agreement with the preceding discussion, both criteria can be written as

$$
\left|T_{(m, n)}-T_{b b}\right| \geq \delta T_{1} \quad \text { and } \quad\left|T_{(m, n)}-T_{a v}\right| \geq \delta T_{2},
$$

where $\delta T_{1}$ and $\delta T_{2}$ are respectively the threshold values of the first and second criteria. 

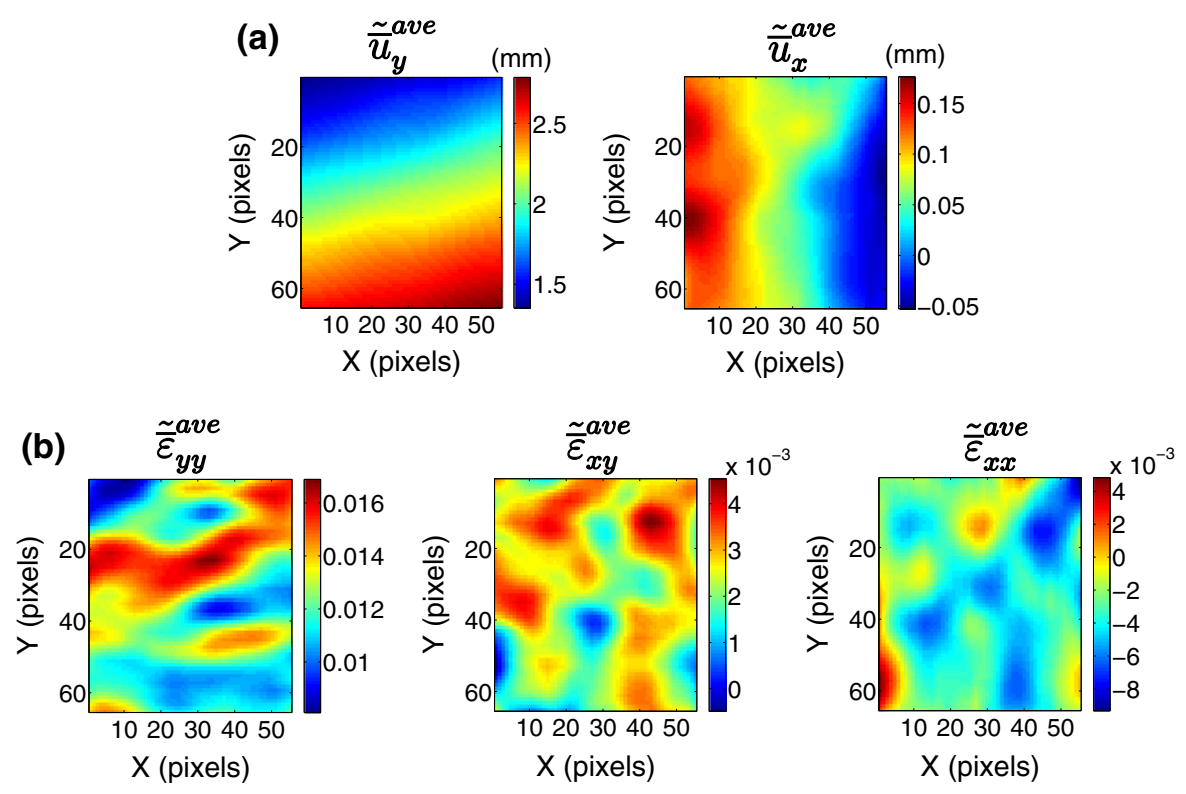

Fig. 6 a Assessed displacement fields in vertical and horizontal directions for $\mathrm{MGF} / 45^{\circ}$ composite. The displacements are expressed in millimeters. b Tensile, shear, and contraction components of Hencky strain for $\mathrm{MGF} / 45^{\circ}$ composite. The spatial resolution was $0.1851 \mathrm{~mm} /$ pixel

The room temperature was taken into account by recording the temperature data of an unloaded specimen (dummy) kept nearby the test specimen. The temperature images were then acquired using Altair software and stored on a computer hard drive. A local least-squares fitting method using an optimized approximation functions set was developed to filter the IR images from the noise [16].

The digital image correlation (DIC) technique is a noncontact strain estimate method that offers the possibility of determining full-field measurements of displacement and deformation at the surface of loaded specimens. It consists of capturing consecutive images of a random surface pattern recorded using a CCD camera. To apply this method, the random pattern (local optical signature of a material surface element) was achieved by spraying the specimen surface with a painted speckle pattern (pulverization of black and white paints). A cold light source was used to obtain well-illuminated, visible, high-contrast images. For the $10 \mathrm{~Hz}$ loading test, the time integration was reduced in such a way as to obtain reliable measurements, thereby avoiding the adverse effects (such as sample heating) related to the use of the light source. The change in surface characteristics of the specimen was evaluated by matching different subcells of two images captured before and after loading the specimen. Because it is almost impossible to find a matched zone using few pixels, a subset with multiple pixel points was defined to perform the matching process. Adequate DIC software was required to obtain information on the positional changes in the surface under investigation. In our case, we used the custom Kelkins software package developed by the ThM2 team of $L M G C$. Displacement fields were thus determined using suitable algorithms following digitization of images captured by the visible camera [29]. Figure 6a shows typical displacement fields (horizontal and vertical directions) generated by the DIC software for an $\mathrm{MGF} / 45^{\circ}$ composite. These kinematic data stand for the mean displacements averaged over 100 cycles and are denoted by $\tilde{\bar{u}}_{y}^{\text {ave }}, \tilde{\bar{u}}_{x}^{\text {ave }}$, corresponding to $10 \mathrm{~s}$ of cyclic loading. Since the experimental data were inherently noisy, appropriate spatial smoothing methods were needed to reduce the effect of noise on the strain estimate. As for the thermal data, we used projections of data on local analytical function in a least-squares sense (1) to approximate the experimental displacement values in the selected regions, (2) to reduce the noise present in these displacements, and (3) to derive the measured strain and strain rate tensors (Fig. 6b).

To determine the energy behavior of the studied composite materials, it is crucial to measure the applied load, temperature, and strain at the same time. Therefore, it is of paramount importance to synchronize the IR and visible 
cameras with the load cell sensor of the hydraulic testing machine (MTS). A specific tailored electronic device called a synchrocam was designed to capture the three data types together. Both imaging systems were then triggered from a digital pulse to capture data concurrently. This ensured that all data captured by the imaging systems and MTS were initiated at the same time. Fortunately, in the current investigation both systems were initiated at the same time with identical frame rates, which facilitated the image processing.

In addition, the geometric transformation between the frames of reference of the two cameras was determined using a calibrated target. The correspondence between the spatial coordinates of the thermal image $\left(x_{\mathrm{IR}}, y_{\mathrm{IR}}\right)$ and those of the visible image $\left(x_{\mathrm{CCD}}, y_{\mathrm{CCD}}\right)$ was established by triggering the motion of the calibrated target using the following equations:

$$
\left(\begin{array}{l}
x_{\mathrm{CCD}} \\
y_{\mathrm{CCD}}
\end{array}\right)=\underbrace{\left(\begin{array}{ll}
\gamma_{x} & \eta_{x} \\
\gamma_{y} & \eta_{y}
\end{array}\right)}_{\mathscr{R}}\left(\begin{array}{l}
x_{\mathrm{IR}} \\
y_{\mathrm{IR}}
\end{array}\right)+\underbrace{\left(\begin{array}{l}
\lambda_{x} \\
\lambda_{y}
\end{array}\right)}_{\mathscr{D}},
$$

where $\mathscr{R}$ is the rotation matrix and $\mathscr{D}$ a displacement vector. This spatial correspondence was carried out before each new test.

\section{Experimental results}

\subsection{Specimens and loading}

All tests were performed on plane composite specimens with a rectangular active gauge section $(12 \mathrm{~mm} \times 10 \mathrm{~mm} \times$ $3.14 \mathrm{~mm}$ ). The considered sample geometry is outlined in Fig. 7a. Cyclic tensile-tensile loads were carried out under a stress ratio of 0.1 , while the loading frequency was equal to $10 \mathrm{~Hz}$. The number of cycles was $10^{3}$ and high enough to raise the kinematic and thermal properties of the investigated composite materials. The mechanical loading is illustrated in Fig. 3b.

\subsection{Strain pattern analysis}

The kinematic assessments were analyzed in terms of mean Hencky strain components. The goal was to track the possible development of strain localization zones throughout the oligocyclic fatigue tests. DIC techniques were systematically used to derive the three components of the in-plane strain tensor. The image processing was performed on the three fiber-reinforced composites. The results shown hereafter (Figs. 8, 9, and 10) correspond to the $10 \mathrm{~Hz}$ cyclic loading tests already mentioned for the three fiber directions.
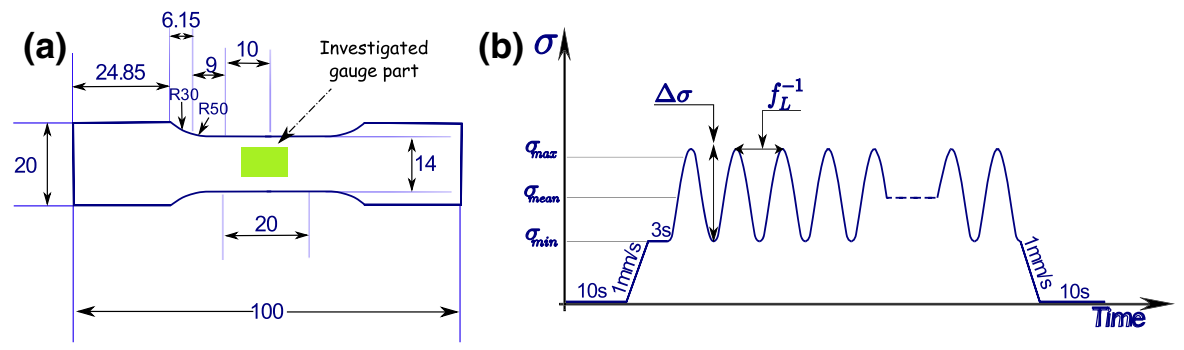

Fig. 7 a Shape of specimens used for experiments (all dimensions are in millimeters) showing relative position of observed gauge part. b Diagram of mechanical loading used in experiments 
- Figure 8 shows five strain fields regularly captured throughout the test for each of the three components of the mean strain tensor $\tilde{\bar{\varepsilon}}^{\text {ave }}$ associated with the $\mathrm{MGF} / 0^{\circ}$ composite. The kinematic patterns indicated a precocious and gradual development of strain localization zones. These mechanisms were clearly visible for the longitudinal and shear components. However, the contraction strain patterns corresponding to the direction perpendicular to loading and fiber orientation were also heterogeneous but remained relatively constant throughout the test. The statistics associated with these different strain distributions are pooled in Table 2. Note that the standard deviation values (SDVs) corresponding to these distributions were of the same order of magnitude as those of their mean values. We stress, however, that these SDVs essentially stem from the material heterogeneity and not from the experimental noise on kinematic data. We thus considered the mean strain values as being representative of the macroscopic deformation state of the composite specimen despite the existence of high material heterogeneity.

- In the same way, Figs. 9 and 10 showed the strain pattern characteristics for MGF $/ 45^{\circ}$ and $\mathrm{MGF} / 90^{\circ}$ composites. The strain localization was again omnipresent from the very beginning of the cyclic tests with marked development particularly for the longitudinal and shear components. The localization patterns also seemed to progress preferably according to the fiber orientation angle.

- Regarding the statistics of the strain distributions in Table 2, a macroscopic contraction ratio was computed. For the three orientation angles it was observed that the contraction ratio was greater for the $0^{\circ}$ direction than that associated with the $90^{\circ}$ angle, as expected from a physical standpoint, and the contraction ratio for a given orientation regularly decreased, as often observed for porous or damaged material.

\subsection{Thermal and calorimetric analysis}

In this section, we focus mainly on how to deal with deformation mechanisms in order to track fiber orientation effects in the investigated composite materials. The analysis was performed using calorimetric investigations based essentially on computing heat sources involved in the investigated gauge part of SFRSP composites. These heat sources could not be inferred from the mechanical characteristics of the composites, but they were assessed using thermal information provided by the IR camera.

So far, many attempts have been made to solve constitutive equations using many simplifications and hypotheses. Because IR cameras only provide temperature distributions at the surface of loaded thin, flat specimens, the present study, which was conducted in the field of composites, assumed plane conditions. Any changes in the off-plane fiber direction have not yet been investigated.

The primary objective of the experimental data processing was to derive the distribution of dissipated energy involved in the investigated gauge part of the materials from the surface temperature measurements. This energy was locally derived from both equations of local heat diffusion and linear thermoelasticity [Eqs. (1) and (6)]. The intrinsic dissipative heat source $\tilde{\overline{\mathcal{D}}}_{1}$ averaged over one complete cycle and divided by $\rho C$ was calculated using the following equation:

$\frac{\tilde{\overline{\mathcal{D}}}_{1}}{\rho C}=f_{L} \int_{(q-1) f_{L}^{-1}}^{q f_{L}^{-1}} \frac{\overline{\mathcal{D}}_{1}(x, y, t)}{\rho C} \mathrm{~d} t$,

where $q$ denotes the cycle number and $f_{L}^{-1}$ the cycling period. $\overline{\mathcal{D}}_{1}$ (respectively $\tilde{\overline{\mathcal{D}}}_{1}$ ) denotes the spatial (and temporal) average(s) of $\mathcal{D}_{1}$ over the sample thickness (and over one complete cycle), which corresponds to the outof-plane axis (Fig. 3). This dissipative term was determined by computing the temporal and spatial differentiation terms, respectively, in Eq. (6) for the heat rate $\partial \bar{\theta} / \partial t$ and the two-dimensional Laplacian $\left(\partial^{2} \bar{\theta} / \partial x^{2}+\partial^{2} \bar{\theta} / \partial y^{2}\right)$. The noise component was mitigated from the data system records (data set) using a local polynomial fitting based on least-squares techniques. These fitting methods were essential for computing reliable estimates of dissipative heat sources but were time consuming and tricky to use. 


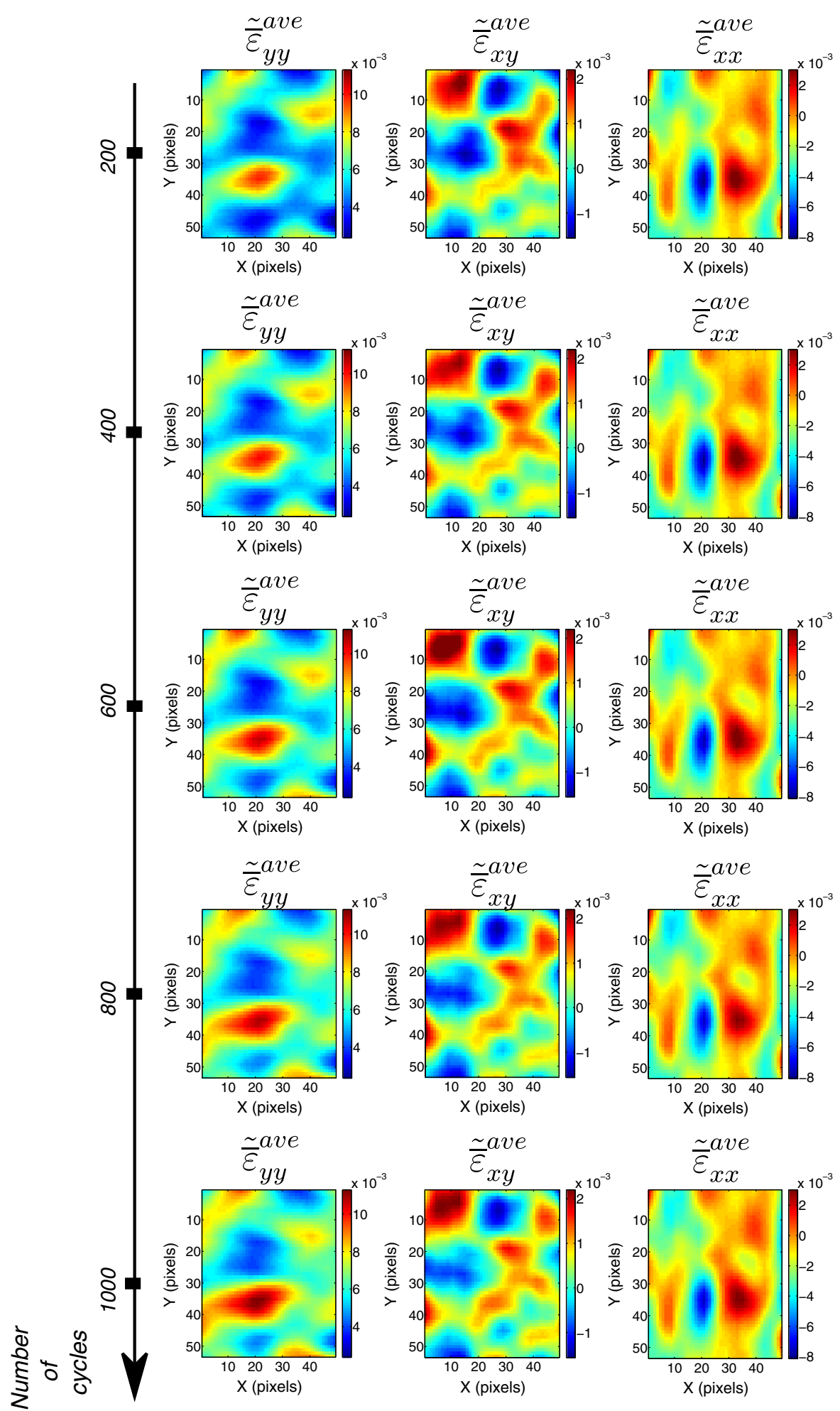

Fig. 8 Tensile, shear, and contraction components of Hencky strain associated with $\mathrm{MGF} / 0^{\circ}$ composite; spatial resolution: $0.1851 \mathrm{~mm} /$ pixel. The standard deviations and spatial averages are summarized in Table 2 

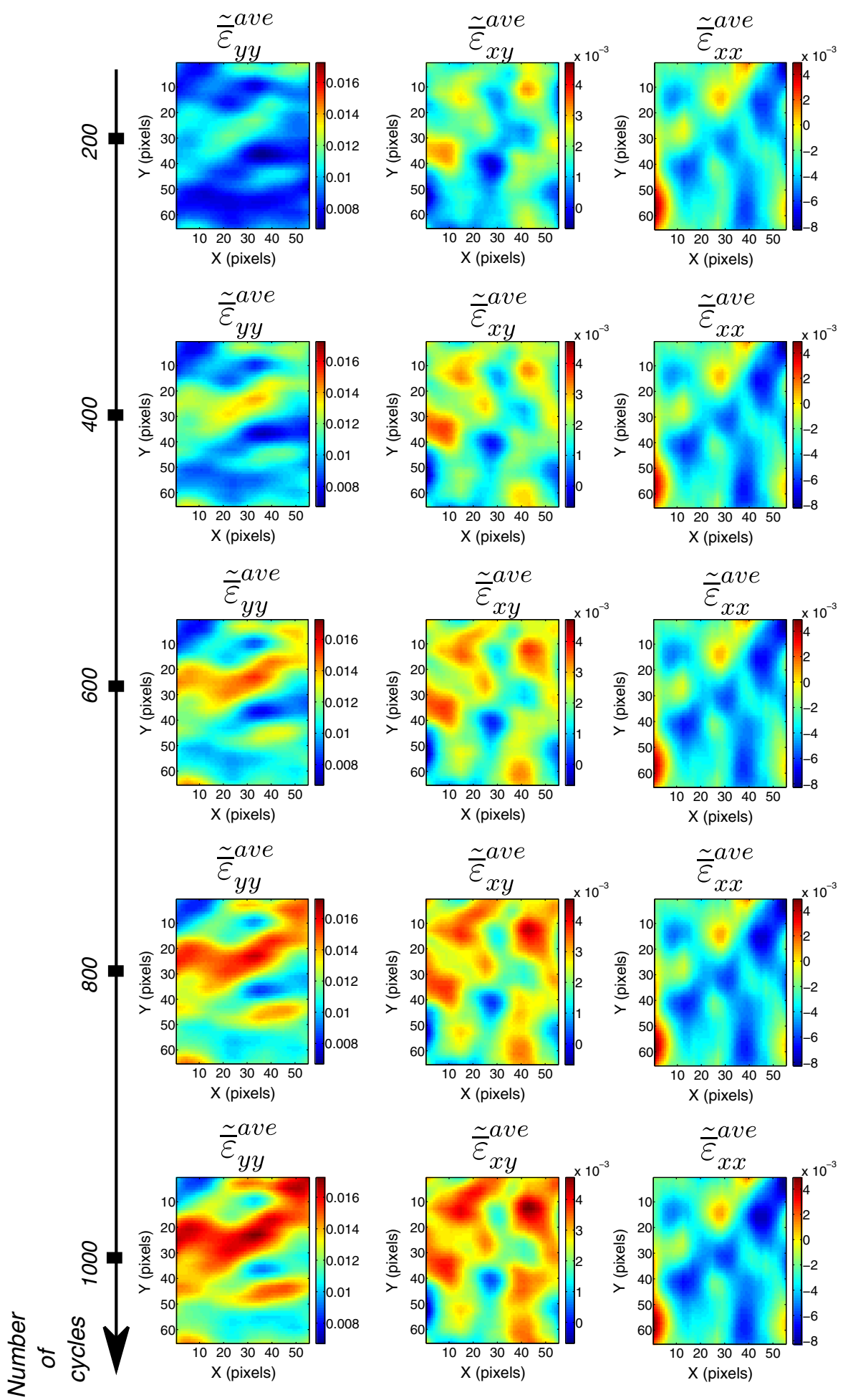

Fig. 9 Tensile, shear, and contraction components of Hencky strain associated with MGF $/ 45^{\circ}$ composite; spatial resolution: $0.1851 \mathrm{~mm} /$ pixel. The standard deviations and spatial averages are summarized in Table 2 


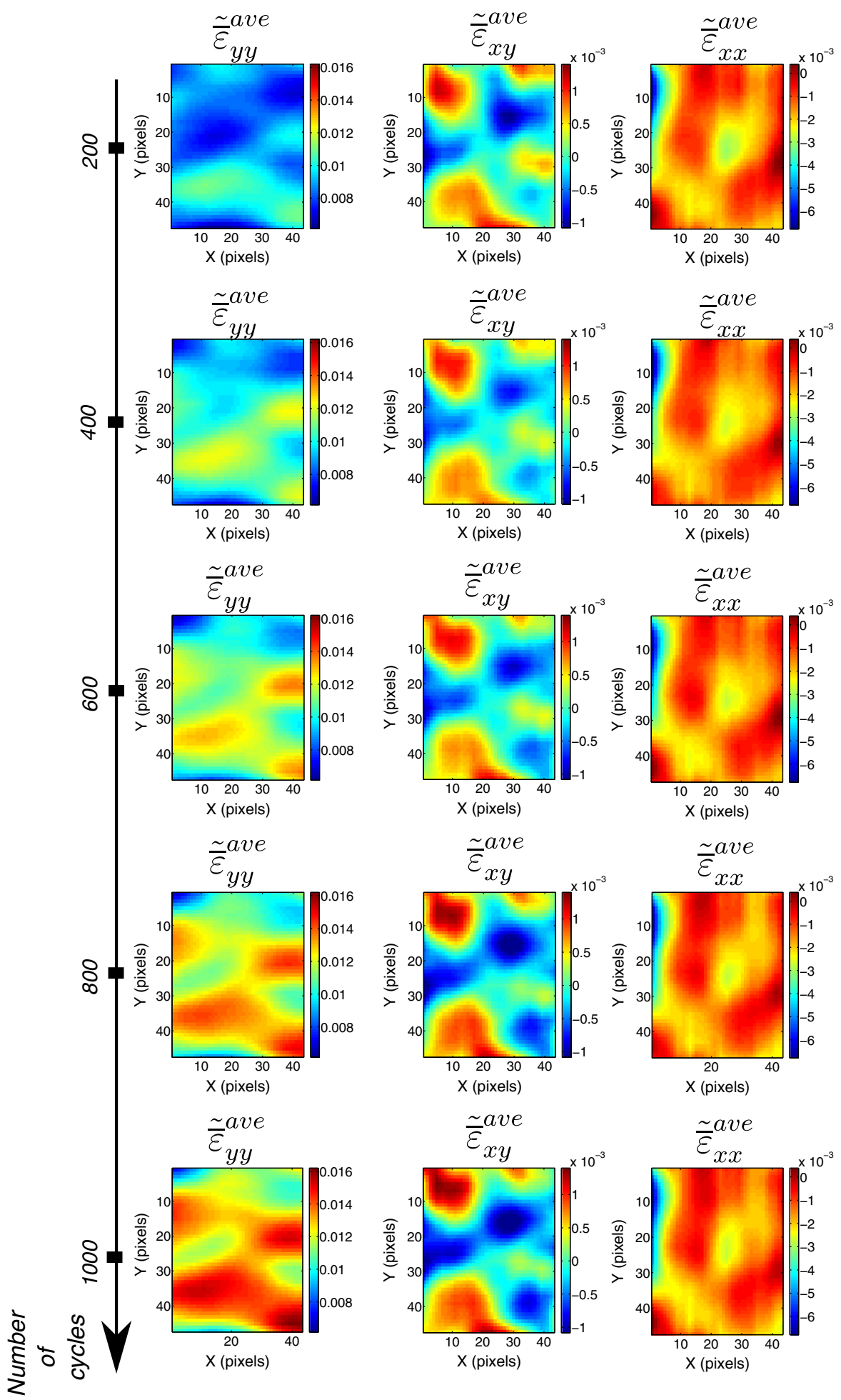

Fig. 10 Tensile, shear, and contraction components of Hencky strain associated with MGF/90 composite; spatial resolution: $0.1851 \mathrm{~mm} /$ pixel. The standard deviations and spatial averages are summarized in Table 2 
Table 2 Standard deviations and spatial averages associated with selected kinematic fields mentioned in Figs. 8, 9, and 10

\begin{tabular}{|c|c|c|c|c|c|c|}
\hline \multirow[t]{2}{*}{ Kinematic data } & \multicolumn{2}{|l|}{$\mathrm{MGF} / 0^{\circ}$} & \multicolumn{2}{|l|}{$\mathrm{MGF} / 45^{\circ}$} & \multicolumn{2}{|l|}{$\mathrm{MGF} / 90^{\circ}$} \\
\hline & SDV & Mean & SDV & Mean & SDV & Mean \\
\hline \multicolumn{7}{|c|}{200 cycles (1st stage) } \\
\hline$\tilde{\bar{\varepsilon}}_{x x}^{\text {ave }}$ & $17 \times 10^{-4}$ & $-16 \times 10^{-4}$ & $17 \times 10^{-4}$ & $-29 \times 10^{-4}$ & $9 \times 10^{-4}$ & $-1.6 \times 10^{-3}$ \\
\hline$\tilde{\bar{\varepsilon}}_{x y}^{\text {ave }}$ & $7.4 \times 10^{-4}$ & $3.3 \times 10^{-4}$ & $6.4 \times 10^{-4}$ & $16 \times 10^{-4}$ & $5 \times 10^{-4}$ & $9.1 \times 10^{-5}$ \\
\hline$\tilde{\bar{\varepsilon}}_{y y}^{\text {ave }}$ & $13 \times 10^{-4}$ & $58 \times 10^{-4}$ & $11 \times 10^{-4}$ & $9.3 \times 10^{-3}$ & $1 \times 10^{-3}$ & $8.9 \times 10^{-3}$ \\
\hline \multicolumn{7}{|c|}{400 cycles ( 2 nd stage) } \\
\hline$\tilde{\bar{\varepsilon}}_{x x}^{\text {ave }}$ & $17 \times 10^{-4}$ & $-16 \times 10^{-4}$ & $18 \times 10^{-4}$ & $-31 \times 10^{-4}$ & $9 \times 10^{-4}$ & $-1.6 \times 10^{-3}$ \\
\hline$\tilde{\overline{\boldsymbol{\varepsilon}}}_{x y}^{\mathrm{ave}}$ & $7.4 \times 10^{-4}$ & $3.5 \times 10^{-4}$ & $6.7 \times 10^{-4}$ & $19 \times 10^{-4}$ & $4 \times 10^{-4}$ & $8.4 \times 10^{-5}$ \\
\hline$\tilde{\bar{\varepsilon}}_{y y}^{\text {ave }}$ & $13 \times 10^{-4}$ & $61 \times 10^{-4}$ & $14 \times 10^{-4}$ & $10.6 \times 10^{-3}$ & $1.2 \times 10^{-3}$ & $10.2 \times 10^{-3}$ \\
\hline \multicolumn{7}{|c|}{600 cycles (3rd stage) } \\
\hline$\tilde{\overline{\boldsymbol{\varepsilon}}}_{x x}^{\text {ave }}$ & $17 \times 10^{-4}$ & $-16 \times 10^{-4}$ & $18 \times 10^{-4}$ & $-32 \times 10^{-4}$ & $9 \times 10^{-4}$ & $-1.6 \times 10^{-3}$ \\
\hline$\tilde{\bar{\varepsilon}}_{x y}^{\text {ave }}$ & $8.1 \times 10^{-4}$ & $3.6 \times 10^{-4}$ & $7.1 \times 10^{-4}$ & $22 \times 10^{-4}$ & $5 \times 10^{-4}$ & $5.8 \times 10^{-5}$ \\
\hline$\tilde{\bar{\varepsilon}}_{y y}^{\text {ave }}$ & $14 \times 10^{-4}$ & $63 \times 10^{-4}$ & $16 \times 10^{-4}$ & $11.5 \times 10^{-3}$ & $1.3 \times 10^{-3}$ & $11.1 \times 10^{-3}$ \\
\hline \multicolumn{7}{|c|}{800 cycles (4th stage) } \\
\hline$\tilde{\bar{\varepsilon}}_{x x}^{\text {ave }}$ & $16 \times 10^{-4}$ & $-16 \times 10^{-4}$ & $19 \times 10^{-4}$ & $-33 \times 10^{-4}$ & $9 \times 10^{-4}$ & $-1.6 \times 10^{-3}$ \\
\hline$\tilde{\overline{\boldsymbol{\varepsilon}}}_{x y}^{\mathrm{ave}}$ & $7.3 \times 10^{-4}$ & $3.8 \times 10^{-4}$ & $7.4 \times 10^{-4}$ & $24 \times 10^{-4}$ & $6 \times 10^{-4}$ & $3.5 \times 10^{-5}$ \\
\hline$\tilde{\bar{\varepsilon}}_{y y}^{\text {ave }}$ & $14 \times 10^{-4}$ & $65 \times 10^{-4}$ & $17 \times 10^{-4}$ & $12.3 \times 10^{-3}$ & $1.4 \times 10^{-3}$ & $12 \times 10^{-3}$ \\
\hline \multicolumn{7}{|c|}{1,000 cycles (5th stage) } \\
\hline$\tilde{\bar{\varepsilon}}_{x x}^{\text {ave }}$ & $16 \times 10^{-4}$ & $-15 \times 10^{-4}$ & $19 \times 10^{-4}$ & $-33 \times 10^{-4}$ & $10 \times 10^{-4}$ & $-1.6 \times 10^{-3}$ \\
\hline$\tilde{\bar{\varepsilon}}_{x y}^{\text {ave }}$ & $7.1 \times 10^{-4}$ & $4 \times 10^{-4}$ & $7.8 \times 10^{-4}$ & $26 \times 10^{-4}$ & $6 \times 10^{-4}$ & $2.5 \times 10^{-5}$ \\
\hline \multirow[t]{3}{*}{$\tilde{\overline{\boldsymbol{\varepsilon}}}_{y y}^{\mathrm{ave}}$} & $14 \times 10^{-4}$ & $67 \times 10^{-4}$ & $19 \times 10^{-4}$ & $13 \times 10^{-3}$ & $1.5 \times 10^{-3}$ & $12.9 \times 10^{-3}$ \\
\hline & \multicolumn{5}{|c|}{ Contraction ratio } & \\
\hline & 1st stage & 2nd stage & 3rd stage & 4th stage & 5th stage & \\
\hline \multicolumn{7}{|l|}{$\mathrm{MGF} / 0^{\circ}$} \\
\hline$\tilde{\overline{\boldsymbol{v}}}$ & 0.276 & 0.262 & 0.254 & 0.236 & 0.224 & \\
\hline \multicolumn{7}{|l|}{$\mathrm{MGF} / 45^{\circ}$} \\
\hline$\tilde{\overline{\boldsymbol{v}}}$ & 0.312 & 0.293 & 0.278 & 0.268 & 0.254 & \\
\hline \multicolumn{7}{|l|}{$\mathrm{MGF} / 90^{\circ}$} \\
\hline$\tilde{\bar{v}}$ & 0.179 & 0.157 & 0.144 & 0.133 & 0.124 & \\
\hline
\end{tabular}

The thermoelastic source ranges $\overline{\Delta s}_{\text {ther }}=\max \left(\bar{s}_{\text {ther }}\right)-\min \left(\bar{s}_{\text {ther }}\right)$ divided by $\rho C$, derived from Eq. (1), were estimated using the expression below:

$\frac{\overline{\Delta s}_{\text {ther }}}{\rho C}=2 \pi f_{L} \overline{\Delta \theta}$,

where $f_{L}$ stands for the loading frequency and $\overline{\Delta \theta}$ the range of the temperature oscillation over a complete cycle.

In what follows, the temperature variations, mean intrinsic dissipation, and thermoelastic source amplitudes were assessed over each complete load-unload cycle. The selected images in Figs. 11, 12, and 13 were processed to allow 
visualization of the thermal and heat source fields during the first few cycles of the loaded specimens. The values of the featured heat sources were depicted and graduated on a color bar. Note that the heat sources were divided by $\rho C$ and thus expressed in degrees Celsius per second in order to define for each type of source an equivalent heating rate, thereby facilitating comparisons between the dissipative and coupling sources.

It should be noted that the distributions of these heat and thermal data were not uniform and varied markedly. The spatial heterogeneities were clearly visible throughout all the selected fatigue steps. By contrast with the polymer matrix, for which it was shown that the localization of dissipative mechanisms took place in an arbitrary direction [14], the propagation of these mechanisms, when the matrix material was reinforced with fibers, seemed to be guided by fibers and travelled along the fiber direction. For instance, the hot spot propagation in $\mathrm{MGF} / 0^{\circ}$ was directed along the specimen length, and hence the hottest area propagated freely without deviation (Fig. 11). In this case, the intrinsic dissipation had the lowest maximum value of $0.12^{\circ} \mathrm{C} \mathrm{s}^{-1}$ compared with that of thermoelasticity, which was $6.5^{\circ} \mathrm{C} \mathrm{s}^{-1}$. Note that the location of hot spots in dissipation fields was not necessarily at the place where the thermoelasticity amplitudes (stress) were at their maximum. This means that the first invariant of the stress tensor was not the only parameter that influenced the dissipative behavior of the material. Fibers could cause shear-stress concentrations at the interfaces (related to the second invariant of the deviatoric stress tensor) where hot spots in thermoelasticity fields were more pronounced, but they could not lead to localized sites at the same hottest zones in dissipation fields. This fiber effect changed completely when the fiber orientation took different angles. For instance, in $\mathrm{MGF} / 45^{\circ}$, the localized hottest areas were directed along the fibers, i.e., $45^{\circ}$. However, in the case of $\mathrm{MGF} / 90^{\circ}$, these hottest areas were diverted along $90^{\circ}$.

Determination of local energy balances was time consuming in terms of data processing time and necessitated a high memory storage capacity. For example, the time computation required for smoothing processes was very high and could exceed many months, depending on the computational resources. We thus decided to study the overall energy evolving using a global approach. This global approach is addressed and discussed in the following subsections.

\subsection{Stress versus strain hysteresis loops}

Cyclic stress-strain curves associated with the tensile-tensile tests of specimens of short-fiber-reinforced PA6.6 are depicted in Fig. 14. The experiments were performed at $10 \mathrm{~Hz}$ loading frequency, 0.1 load ratio, and 1,000 cycles.

The in-plane deformation components $\bar{\varepsilon}_{y y}, \bar{\varepsilon}_{x y}$, and $\bar{\varepsilon}_{x x}$ were determined using DIC techniques. The applied mechanical load $f$ was measured via the load cell of the hydraulic testing machine. The tensile, shear and contraction components of the stress tensor were derived from the equilibrium equations based on several assumptions (e.g., plane stress pattern, material incompressibility, no overall shear loading and lateral surfaces of the specimen free of stress [29]):

$\bar{\sigma}_{y y}(y, t)=\frac{f(t)}{S_{o}} \exp \left(\bar{\varepsilon}_{y y}(y, t)\right)$,

$\bar{\sigma}_{x y}(x, y, t)=-\bar{\sigma}_{y y}(y, t) \frac{\partial \bar{\varepsilon}_{y y}(y, t)}{\partial y} x$,

$\bar{\sigma}_{x x}(x, y, t)=\frac{\partial}{\partial y}\left(\frac{\bar{\sigma}_{y y}(y, t)}{2} \frac{\partial \bar{\varepsilon}_{y y}(y, t)}{\partial y}\right)\left(\frac{l^{2}(y, t)}{4}-x^{2}\right)$,

where $f$ represents the load, $\bar{\varepsilon}_{y y}$ the longitudinal component of Hencky strain and $l, S_{0}$ are respectively the width and the initial cross-section of the sample.

The mechanical responses exhibited anisotropic elasticity due to the fiber orientation. According to the slopes of the hysteresis loops (black dashed lines), it seemed that the composite with fibers oriented at $0^{\circ}\left(\mathrm{MGF} / 0^{\circ}\right)$ was more rigid than the other materials. The hysteresis loops were mechanically not stabilized, and no elastic or plastic shakedown was observed. Indeed, all the stress-strain curves did not converge at a limit loop after a high number of cycles (i.e., no clear cyclic stabilization). The ratcheting phenomenon characterized by the shifting of hysteresis 


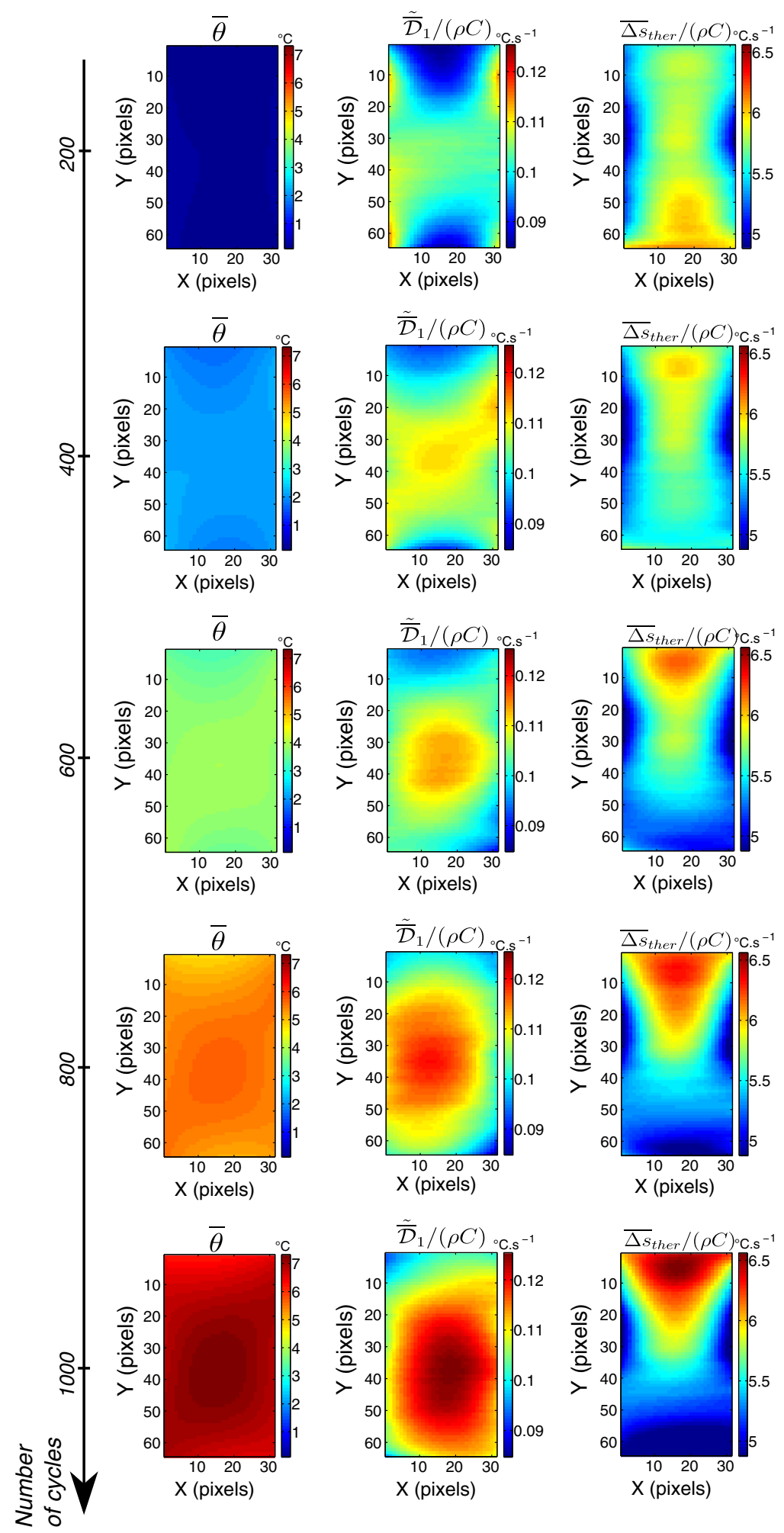

Fig. 11 Fields of temperature variations, mean intrinsic dissipation per cycle, and thermoelastic source amplitudes of a PA6.6 specimen reinforced with $30 \%$ of short glass fibers oriented at $0^{\circ}$; spatial resolution: $0.357 \mathrm{~mm} /$ pixel 


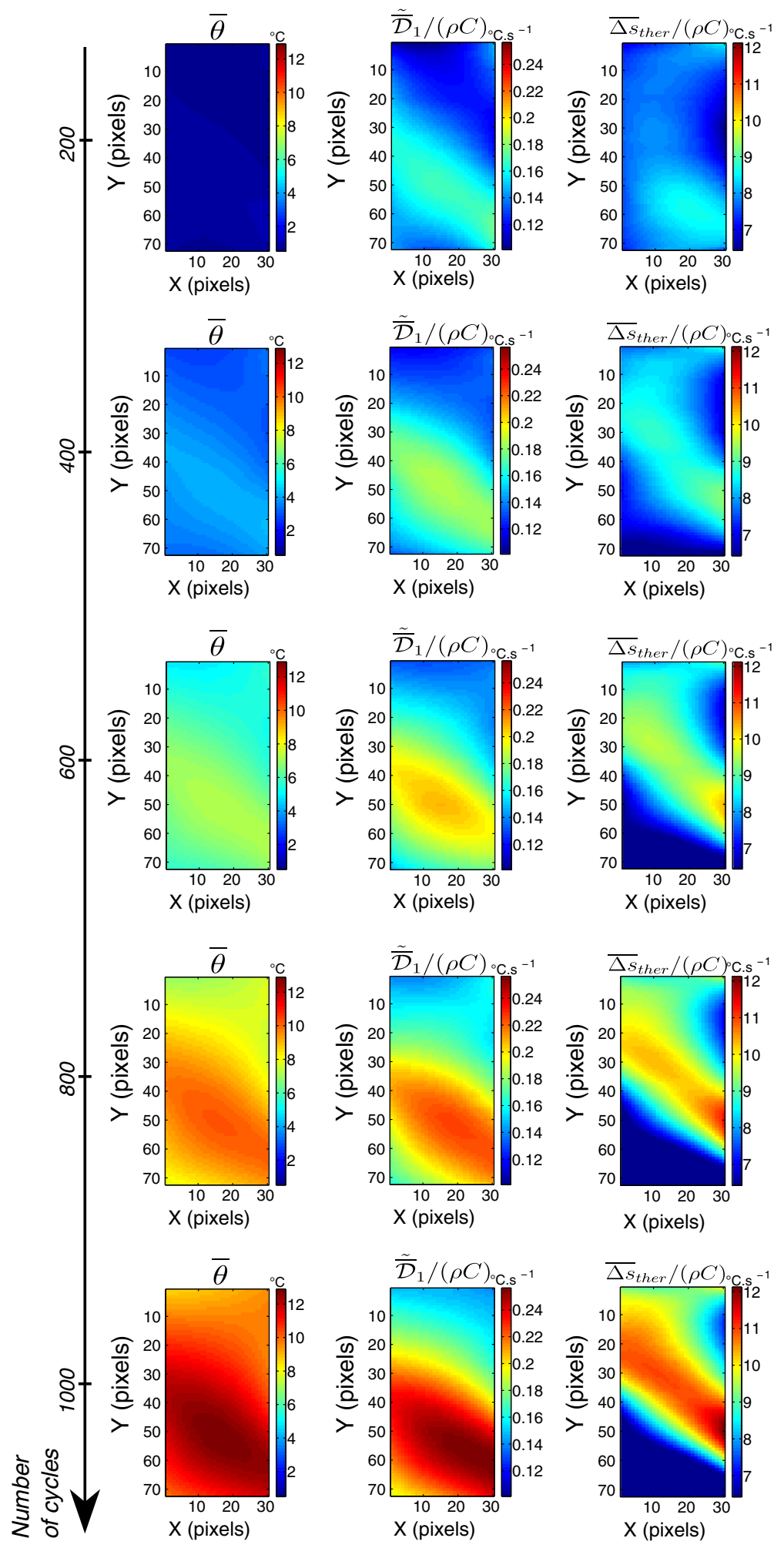

Fig. 12 Fields of temperature variations, mean intrinsic dissipation per cycle, and thermoelastic source amplitudes of a PA6.6 specimen reinforced with $30 \%$ of short glass fibers oriented at $45^{\circ}$ 


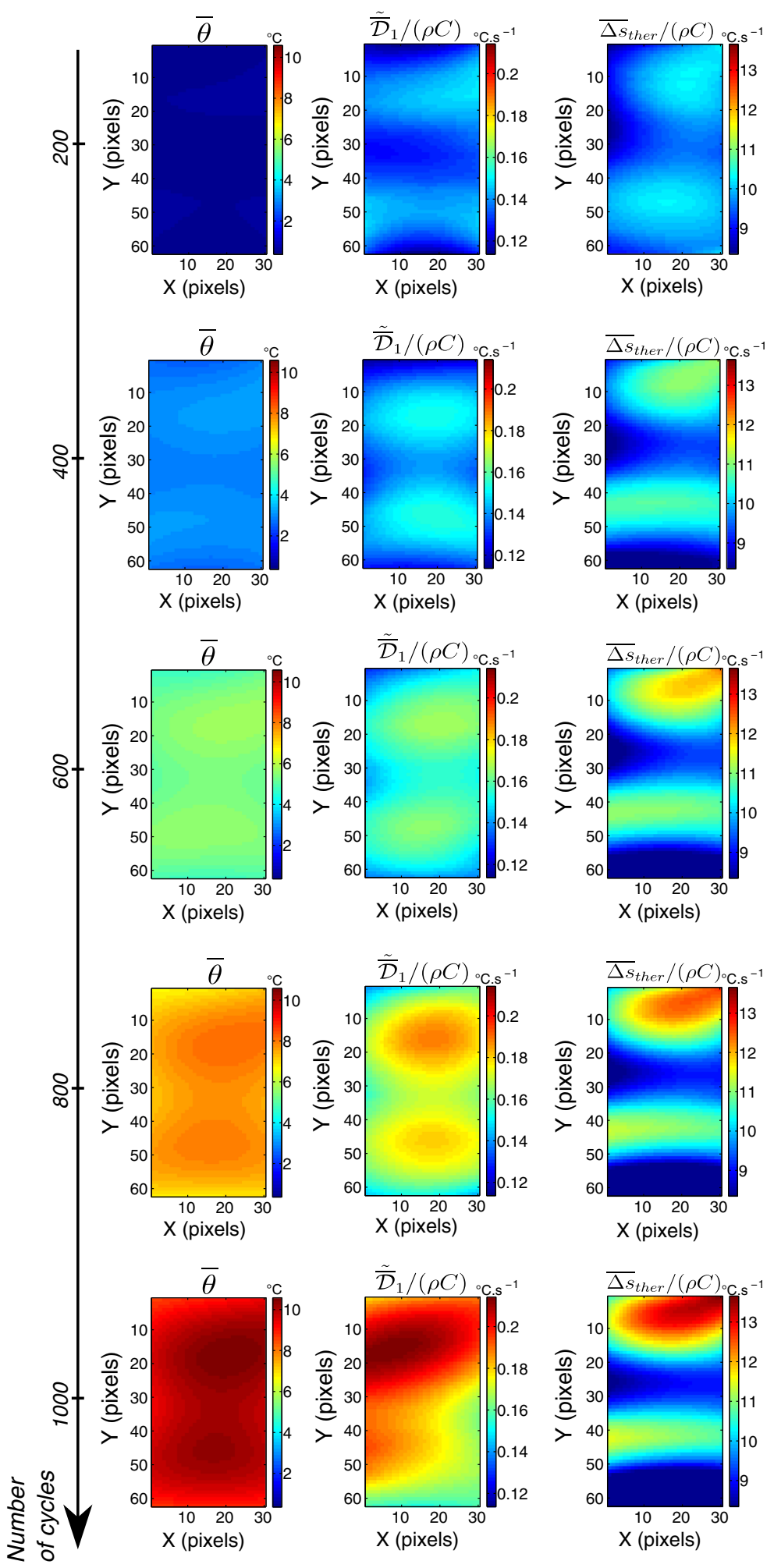

Fig. 13 Fields of temperature variations, mean intrinsic dissipation per cycle, and thermoelastic source amplitudes of a PA6.6 specimen reinforced with $30 \%$ of short glass fibers oriented at $90^{\circ}$ 

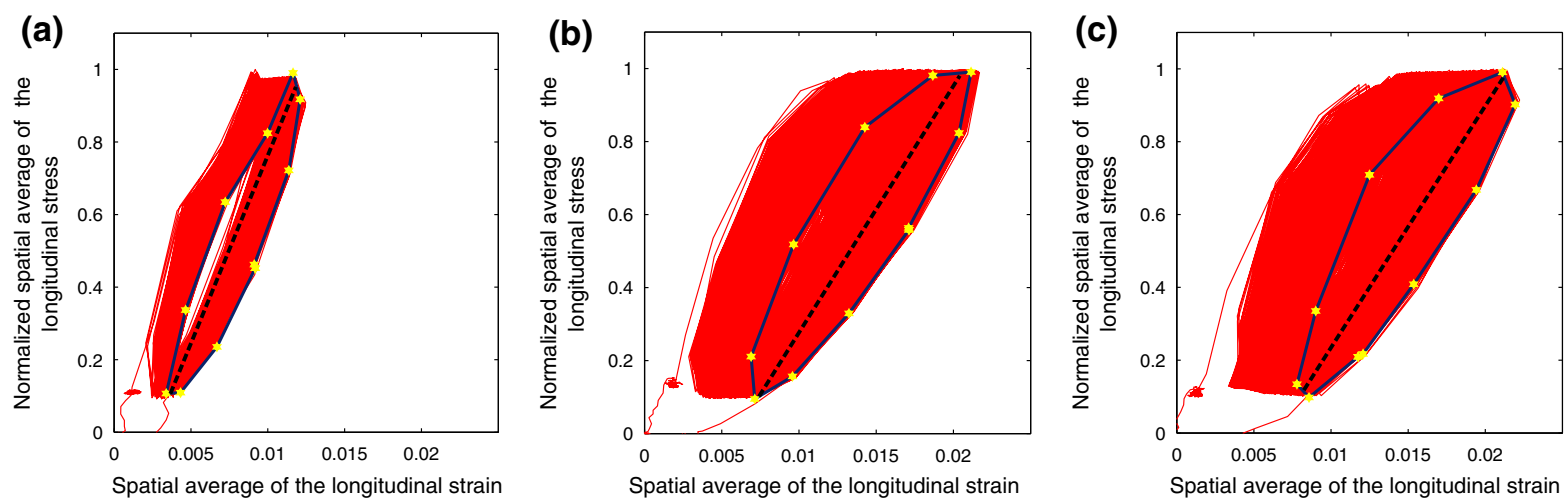

Fig. 14 Normalized stress versus longitudinal strain hysteresis loops associated with tensile-tensile loading of the investigated a $\mathrm{MGF} / 0^{\circ}, \mathbf{b} \mathrm{MGF} / 45^{\circ}$ and $\mathbf{c} \mathrm{MGF} / 90^{\circ}$ specimens

loops was less pronounced in $\mathrm{MGF} / 0^{\circ}$, whereas it was significant in $\mathrm{MGF} / 45^{\circ}$ and $\mathrm{MGF} / 90^{\circ}$. Note also that loops associated with the $\mathrm{MGF} / 0^{\circ}$ were much thinner compared to those in $\mathrm{MGF} / 45^{\circ}$ and $\mathrm{MGF} / 90^{\circ}$.

As it is often recognized that hysteresis loops offer useful information on the material state time course, it was very necessary to gain further insight from a thermodynamic standpoint and characterize the energy content of the ratcheting behavior associated with the investigated materials. It was necessary to determine the parameters upon which the nature of strain accumulation depends. These points are addressed by drawing up energy balances in the next subsection.

\subsection{Energy balances using “0D” approach}

By combining both IR and digital imaging techniques, we drew up local energy rate balances. We measured all energy rates that were involved in the fatigue behavior of specimens of short-fiber-reinforced PA6.6. Different fiber configurations were systematically considered: $0^{\circ}, 45^{\circ}$, and $90^{\circ}$. The energy rates as a function of the cycle number were plotted in Fig. 15a, c, e. The deformation energy rate per cycle was obtained from the hysteresis area $\left\langle\tilde{\mathcal{A}}_{\text {hys }}\right\rangle$ enclosed in the stress-strain loop and the loading frequency using the plane stress and small deformation hypotheses:

$\left\langle\tilde{\mathcal{W}}_{\text {def }}\right\rangle=f_{L}\left\langle\tilde{\mathcal{A}}_{\text {hys }}\right\rangle=f_{L} \int_{(q-1) f_{L}^{-1}}^{q f_{L}^{-1}} \overbrace{\left\langle\sigma_{y y}\right\rangle\left\langle\dot{\varepsilon}_{y y}\right\rangle+\underbrace{2\left\langle\sigma_{x y}\right\rangle\left\langle\dot{\varepsilon}_{x y}\right\rangle+\left\langle\sigma_{x x}\right\rangle\left\langle\dot{\varepsilon}_{x x}\right\rangle}_{\text {negligible products }}}^{\text {plane decomposition }} \mathrm{d} t$.

The shear and contraction products were negligible over the test duration, and only the tensile product was considered in the plane decomposition of the mechanical energy rate. The values of this energy rate per cycle were then compared with those of the mean intrinsic dissipation per cycle to estimate the change in stored energy over the first few deformation cycles. The mean values of these dissipative heat sources were deduced from the integrated form of the heat equation over the observable gauge part, given by

$\left\langle\tilde{\mathcal{D}}_{1}\right\rangle=\rho C f_{L} \int_{(q-1) f_{L}^{-1}}^{q f_{L}^{-1}}\left(\left\langle\frac{\mathrm{d} \theta}{\mathrm{d} t}\right\rangle+\left\langle\frac{\theta}{\tau_{\mathrm{th}}^{0 \mathrm{~d}}}\right\rangle\right) \mathrm{d} t$.

The time constant $\tau_{\text {th }}^{\text {Od }}$ characterizes the overall heat losses. It depends strongly on the sample geometry, which changed during the test. In Eq. (16), the spatial average $\left\langle\tilde{\mathcal{D}}_{1}\right\rangle$ was assumed to be representative of the dissipative heat sources over the investigated gauge part and the cycle $q$. This allowed us not only to mitigate the noise level but also to reduce the time consumption.

The values of these energy data as a function of the cycling number are reported in Fig. 15a, c, e. The amount of heat locally generated by strain was related by the heat conversion factor, i.e., the Taylor-Quinney coefficient 
(a)

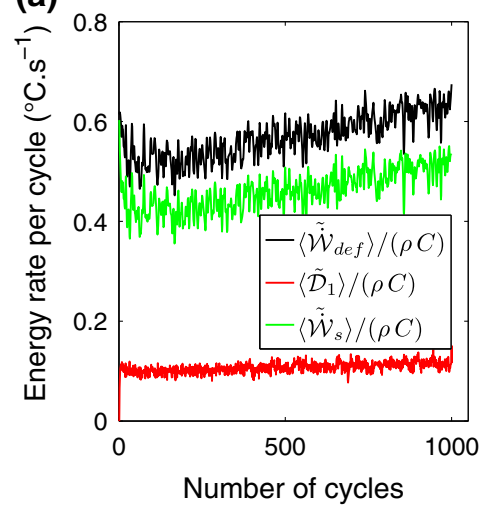

(b)

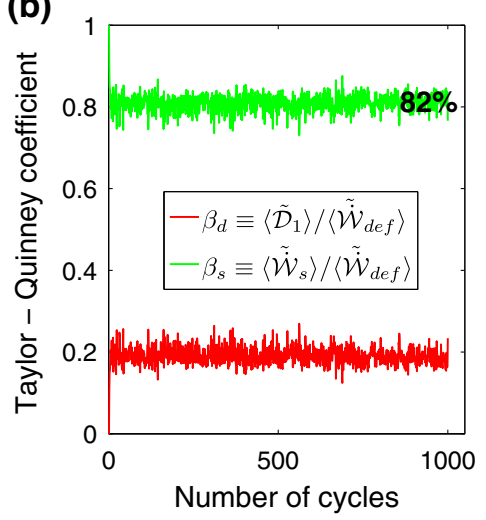

(c)

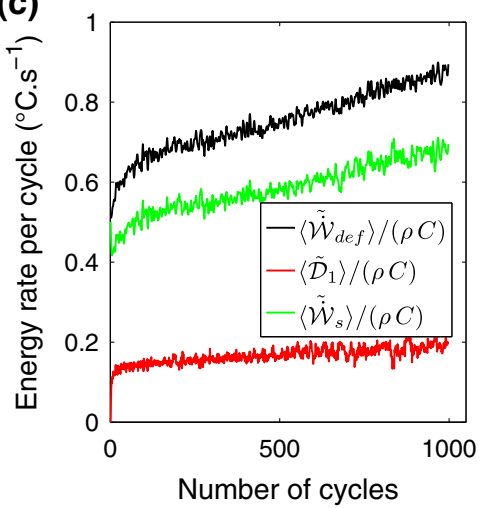

(d)

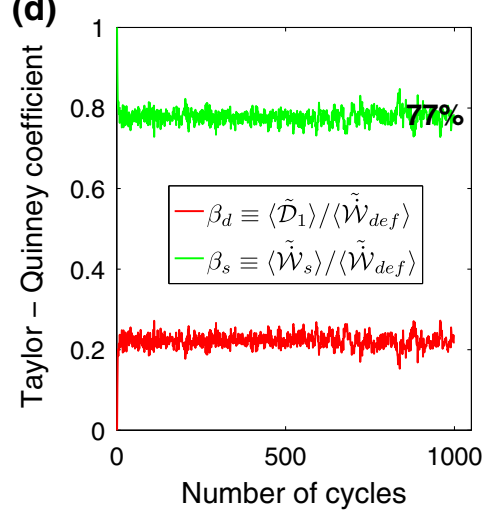

(e)

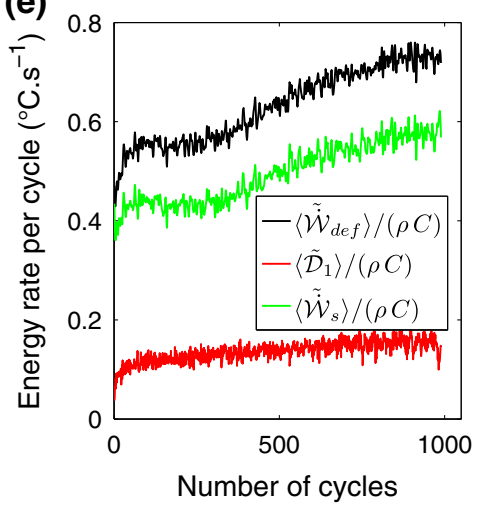

(f)

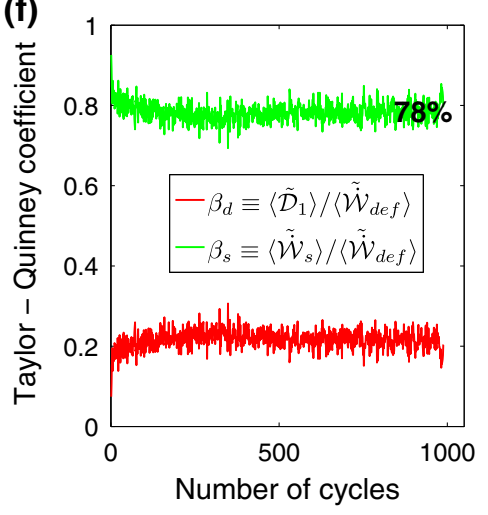

Fig. 15 Energy balance for the investigated fiber configurations a MGF/ $/ 0^{\circ}, \mathbf{c} \mathrm{MGF} / 45^{\circ}$ and $\mathbf{e} \mathrm{MGF} / 90^{\circ}$, and associated Taylor-Quinney coefficients b $\mathrm{MGF} / 0^{\circ}, \mathbf{d} \mathrm{MGF} / 45^{\circ}$ and $\mathbf{f} \mathrm{MGF} / 90^{\circ}$

(Fig. 15b, d, f [30]). This coefficient develops a direct connection with heat sources involved during the loading of materials without establishing their thermomechanical model. It is generally smaller than unity, but it may exceed unity near the final rupture of some materials, where the dissipated energy rate per cycle values exceed those of the deformation energy rate per cycle [14,31,32].

The analysis of these data could shed some light on the energy behavior of these composite materials, especially at very low cycle fatigue. The deformation energy rate was not equal to the rate of heat developed during the cycles. This confirmed that there were internal energy changes within the material and confirmed that the hysteresis loops were not thermodynamically stabilized, i.e., the existence of an internal energy variation. Indeed, the dissipated energy rates per cycle never reached the mechanical energy rates; they differed by as much as fivefold. Otherwise, the largest values of the stored energy rate did not differ between materials. The stored energy rate ratio had approximately the same values in all the investigated materials, i.e., 82,77 , and $78 \%$ for $\mathrm{MGF} / 0^{\circ}, \mathrm{MGF} / 45^{\circ}$, and $\mathrm{MGF} / 90^{\circ}$, respectively.

\section{Conclusions}

The effect of fiber orientation on deformation mechanisms for short-fiber-reinforced semicrystalline-polymeric (SFRSP) composites subjected to tensile-tensile loading tests was experimentally studied. The overall results indicated that the heat source patterns, for example, mean intrinsic dissipation per cycle and thermoelasticity ranges, of these composites closely depended on the fiber orientation angles. The spatial heterogeneities were clearly visible throughout the selected fatigue steps and the localized hottest areas were diverted and directed along the fiber angles, i.e., $0^{\circ}, 45^{\circ}$, and $90^{\circ}$. 
In addition, it was found that there was anisotropic elasticity due to the fiber orientation, and no mechanical or thermodynamic cyclic stabilities were observed. From a mechanical viewpoint, there was neither elastic nor anelastic shakedown, whereas cyclic strain accumulation was observed. From a thermodynamic standpoint, it was shown that the energy content of hysteresis loops was mainly derived from stored $(\approx 80 \%)$ and dissipated $(\approx 20 \%)$ energies. This reflected the significant contribution of the stored energy associated cycle by cycle with the microstructural changes within composite materials.

These imaging techniques have attracted increased attention, and they are still used in subsequent investigations. The objective of earlier investigations was to collect more experimental results and obtain further insight into the PA6.6 cyclic behavior. Future studies will focus on documenting the influence of the hygrothermal environment, over a wide range of relative humidities, $\mathcal{R} \mathcal{H} 0, \mathcal{R} \mathcal{H} 50$, and $\mathcal{R} \mathcal{H} 80$, under two different loading frequencies, $f_{L}=1$ and $10 \mathrm{~Hz}$, on the trend in the energy rate balances associated with the oligocyclic fatigue tests of the PA6.6 matrix. The results would be of substantial interest, especially to those seeking to determine the impact of relative humidity on the constitutive behavior of the PA6.6 matrix and to draw up energy fatigue criteria for the design of industrial composite parts.

Acknowledgments The authors gratefully acknowledge Solvay Engineering Plastics for supporting this work and for providing material data and specimens. This work benefited from the financial support of the French Ministry for Research (ANRT) and was performed in the framework of the European DURAFIP project.

\section{References}

1. Andriyana A, Billon N, Silva L (2010) Mechanical response of a short fiber reinforced thermoplastic: experimental investigation and continuum mechanical modeling. Eur J Mech A Solids 29:1065-1077

2. Launay A, Maitournam MH, Marco Y, Raoult I, Szmytka F (2011) Cyclic behaviour of short glass fibre reinforced polyamide: experimental study and constitutive equations. Int J Plast 27:1267-1293

3. Oliferuk W, Pgadaj S, Grabski M (1985) Energy storage during the tensile deformation of Armco iron and austenitic steel. Mater Sci Eng 70:131-141

4. Chrysochoos A, Maisonneuve O, Martin G, Caumon H, Chezeaux JC (1989) Plastic and dissipated work and stored energy. Nucl Eng Design 114:323-333

5. Rosakis P, Rosakis AJ, Ravichandran G, Hodowany J (2000) Thermodynamic internal variable model for the partition of plastic work into heat and stored energy in metals. J Mech Phys Solids 48:581-607

6. Louche H, Tabourot L (2004) Experimental energetic balance associated to the deformation of an aluminum multicrystal and monocrystal sheet. Mater Sci Forum 467:1395-1400

7. Oliferuk W, Maj M (2009) Stress-strain curve and stored energy during uniaxial deformation of polycrystals. Eur J Mech A Solids 28:266-272

8. Adams GW, Farris RJ (1988) Latent energy of deformation of bisphenol A polycarbonate. J Polym Sci Part B Polym Phys 26:433445

9. Hasan O, Boyce M (1993) Energy storage during inelastic deformation of glassy polymers. Polymer 34:5085-5092

10. Rittel D (1999) On the conversion of plastic work to heat during high strain rate deformation of glassy polymers. Mech Mater 31:131-139

11. Arif MF, Meraghni F, Chemisky Y, Despringre N, Robert G (2014) In situ damage mechanisms investigation of PA66/GF30 composite: effect of relative humidity. Compos Part B 58:487-495

12. Chrysochoos A (2012) Thermomechanical analysis of the cyclic behavior of materials. Procedia IUTAM 4:15-26

13. Chrysochoos A, Wattrisse B, Muracciole JM, EL Kaïm Y (2009) Fields of stored energy associated with localized necking of steel. J Mech Mater Struct 4:245-262

14. Benaarbia A, Chrysochoos A, Robert G (2014) Kinetics of stored and dissipated energies associated with cyclic loadings of dry polyamide 6.6 specimens. Polym Test 34:155-167

15. Louche H, Chrysochoos A (2001) Thermal and dissipative effects accompanying Lüders band propagation. Mater Sci Eng A 307:15-22

16. Boulanger T, Chrysochoos A, Mabru C, Galtier A (2004) Calorimetric analysis of dissipative and thermoelastic effects associated with the fatigue behavior of steels. Int J Fatigue 26:221-229

17. Berthel B, Wattrisse B, Chrysochoos A, Galtier A (2007) Thermographic analysis of fatigue dissipation properties of steel sheets. Strain 43:273-279

18. Thomson W (1853) On the dynamical theory of heat. Trans R Soc 20:261-283

19. Halphen B, Nguyen Qs (1975) On the generalized standards materials (in French). Journal de Mécanique 14:39-63 
20. Germain P (1973) Thermodynamics continuum mechanics (in French). Congrés de Mécanique de Poitiers

21. Lubliner J (1990) Plasticity theory. Ser. Maxwell Macmillan international editions in engineering. Macmillan Publishing, New York

22. Maugin GJ (1992) The thermomechanics of plasticity and fracture, 1st edn. Cambridge University Press, Cambridge, MA

23. Fremond M (2001) Non-smooth thermomechanics. Ser. Physics and astronomy online library. Springer, Berlin

24. Louche H (1999) Infrared thermographic analysis of dissipative effects of localization on steels (in French). University of Montpellier II, Sciences et Techniques du Languedoc, Thesis

25. Berthel B (2007) Dissipation field measurements accompanying the high cycle fatigue of steel by infrared thermography (in French). University of Montpellier II, Sciences et Techniques du Languedoc, Thesis

26. Berthel B, Chrysochoos A, Wattrisse B, Galtier A (2008) Infrared image processing for the calorimetric analysis of fatigue phenomena. Exp Mech 48:79-90

27. Chrysochoos A, Huon V, Jourdan F, Muracciole JM, Peyroux R, Wattrisse B (2010) Use of full-field digital image correlation and infrared thermography measurements for the thermomechanical analysis of material behaviour. Strain 46:117-130

28. Honorat V, Moreau S, Muracciole JM, Wattrisse B, Chrysochoos A (2005) Calorimetric analysis of polymer behaviour using a pixel calibration of an IRFPA camera. Qirt J 2:153-171

29. Wattrisse B, Chrysochoos A, Muracciole JM, Némoz-Gaillard M (2001) Kinematic manifestations of localisation phenomena in steels by digital image correlation. Eur J Mech 20:189-211

30. Taylor GI, Quinney H (1934) The latent energy remaining in a metal after cold working. Proc R Soc A 143:307-326

31. Dillon OWJ (1966) The heat generated during the torsional oscillations of copper tubes. Int J Solids Struct 2:181-204

32. Oliferuk W, Korbel A, Bochniak W (2001) Energy balance and macroscopic strain localization during plastic deformation of polycrystalline metals. Mater Sci Eng A 319:250-253 